\title{
On Closed-Form Solutions to the Position Analysis of Baranov Trusses
}

\author{
Nicolás Rojas, Federico Thomas* \\ Institut de Robòtica i Informàtica Industrial (CSIC-UPC) \\ Llorens Artigas 4-6, 08028 Barcelona, Spain
}

\begin{abstract}
The exact position analysis of a planar mechanism reduces to compute the roots of its characteristic polynomial. Obtaining this polynomial usually involves, as a first step, obtaining a system of equations derived from the independent kinematic loops of the mechanism. Although conceptually simple, the use of kinematic loops for deriving characteristic polynomials leads to complex variable eliminations and, in most cases, trigonometric substitutions. As an alternative, a method based on bilateration has recently been shown to permit obtaining the characteristic polynomials of the three-loop Baranov trusses without relying on variable eliminations or trigonometric substitutions. This paper shows how this technique can be applied to solve the position analysis of all catalogued Baranov trusses. The characteristic polynomials of them all have been derived and, as a result, the maximum number of their assembly modes has been obtained. A comprehensive literature survey is also included.
\end{abstract}

Keywords: Position analysis, Baranov trusses, bilateration, characteristic polynomial.

\section{Introduction}

A non-overconstrained linkage with zero-mobility from which an Assur group can be obtained by removing any of its links is defined as an Assur kinematic chain, basic truss [1, 2], or Baranov truss when no slider joints are considered [3]. Hence, a Baranov truss, named after the Russian kinematician G.G. Baranov who first presented the idea of this kind of truss in 1952 [4], corresponds to multiple Assur groups. The relevance of the Baranov trusses derive from the fact that, if the position analysis of a Baranov truss is solved, the same process can be applied to solve the position analysis of all its corresponding Assur groups. Baranov, in his seminal paper, presented 3 trusses of 7 links and 26 trusses of 9 links. In 1971, Manolescu and Erdelean identified two additional trusses of 9 links that were missing in the initial classification [5], thus completing the classification of Baranov trusses with up to 4 loops. In 1994, Yang and Yao found that the number of Baranov trusses with 11 links is 239 [6]. Unfortunately, their topologies

\footnotetext{
*Corresponding author. Tel.: +34 934015757; fax: +34 934015750.

Email addresses: nrojas@iri.upc.edu (Nicolás Rojas), fthomas@iri.upc.edu (Federico Thomas) Preprint submitted to Mechanism and Machine Theory 
were not made available and, to the best of our knowledge, they have not been published yet. Thus, strictly speaking, only the Baranov trusses with up to 9 links have been catalogued (see Table 1). This catalogue appears in Fig. 1 where each truss is identified using the nomenclature suggested by Manolescu [7].

\begin{tabular}{|c|c|c|c|c|c|c|c|}
\hline \multirow[t]{2}{*}{ Links } & \multirow[t]{2}{*}{ Loops } & \multirow{2}{*}{$\begin{array}{c}\text { Baranov } \\
\text { trusses }\end{array}$} & \multirow{2}{*}{$\begin{array}{l}\text { Available } \\
\text { topology }\end{array}$} & \multicolumn{3}{|c|}{ Coupling degree } & \multirow{2}{*}{$\begin{array}{l}\text { Resulting } \\
\text { Assur groups }\end{array}$} \\
\hline & & & & 1 & 2 & 3 & \\
\hline 3 & 1 & 1 & Yes & 1 & 0 & 0 & 1 \\
\hline 5 & 2 & 1 & Yes & 1 & 0 & 0 & 2 \\
\hline 7 & 3 & 3 & Yes & 3 & 0 & 0 & 10 \\
\hline 9 & 4 & 28 & Yes & 24 & 4 & 0 & 173 \\
\hline 11 & 5 & 239 & No & 197 & 42 & 0 & 5442 \\
\hline 13 & 6 & $?$ & No & $?$ & $?$ & $?$ & 251638 \\
\hline
\end{tabular}

Table 1: Number of Baranov trusses as a function of the number of links (alternatively, number of loops), indication of whether the topologies are available in the literature, number of trusses with different coupling degrees, and number of different Assur groups resulting from eliminating one link from the trusses in each class.

The position analysis problem for a planar truss consists in, given the dimensions of all links, calculating all relative possible transformations between them all. This analysis is usually reduced to finding the roots of a polynomial in one variable, the characteristic polynomial of the truss. When this polynomial is obtained, it is said that the problem is solved in closed form. This approach is usually preferred to numerical approaches because the degree of the polynomial specifies the greatest possible number of assembly configurations of the linkage and modern software provides guaranteed and fast computation of all real roots of a polynomial equation and hence of all assembly configurations of the analyzed linkage.

The closed-form solution to the position analysis of the catalogued Baranov trusses is known for 22 of them. They have been solved on an ad hoc basis by several authors (see Tables 2,11 and the references therein) using mainly elimination techniques, as those based on Sylvester or Dixon resultants, applied to vector loop equations expressed in trigonometric or complex number terms. To the best of our knowledge, the closed-form position analysis of the trusses identified as $9 / B_{2}, 9 / B_{3}, 9 / B_{4}, 9 / B_{5}, 9 / B_{6}, 9 / B_{8}, 9 / B_{9}$, $9 / B_{13}, 9 / B_{18}, 9 / B_{19}$, and $9 / B_{22}$ has not been reported in the literature. Nevertheless, the number of assembly modes of these trusses was studied in [8] using homotopy continuation. However, as it will be shown later, some of the reported results are erroneous. Beyond four loops, the closed-form position analysis of only two 11-link Baranov trusses and one 13-link Baranov truss have been reported. In [9], Lösch solved the five-loop version of the $9 / B_{1}$ Baranov truss with a procedure based on vector method and Gröbner basis. The same truss was analyzed by Wohlhart using Sylvester elimination [10]. Recently, Rojas and Thomas solved the five-loop and six-loop versions of the $9 / B_{10}$ Baranov truss [11].

An $n$-ary link in a Baranov truss introduces a set of distance constraints between the $n$ involved joints. This translates into a set of quadratic equations from which an eliminant can be obtained to get a single equation in one variable. In this case, each equation is simple but the elimination process involve a large number of equations. A more compact elimination process is obtained when the set of $2 n+1$ independent loop 


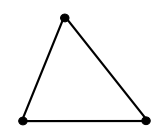
$3 / B_{1}$

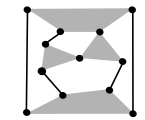

$9 / B_{3}$

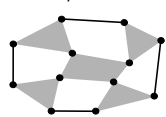

$9 / B_{10}$

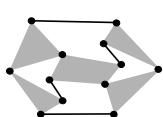

$9 / B_{17}$

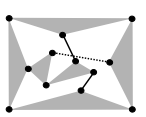

$9 / B_{24}$

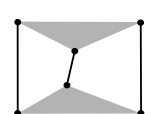

$5 / B_{1}$

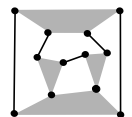

$9 / B_{4}$

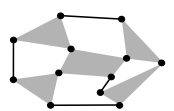

$9 / B_{11}$

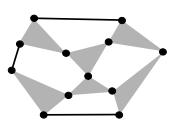

$9 / B_{18}$

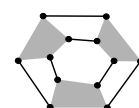

$9 / B_{25}$

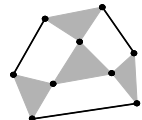

$7 / B_{1}$

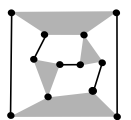

$9 / B_{5}$

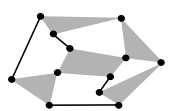

$9 / B_{12}$

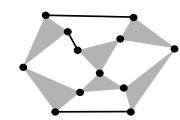

9/B 19

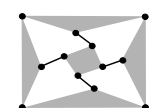

$9 / B_{26}$

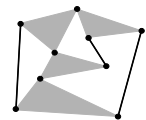

$7 / B_{2}$

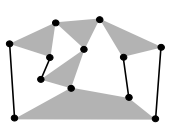

$9 / B_{6}$

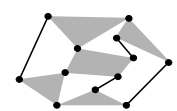

$9 / B_{13}$

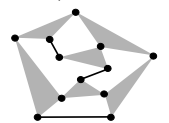

$9 / B_{20}$

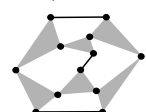

$9 / B_{27}$

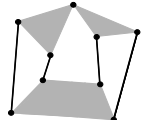

$7 / B_{3}$

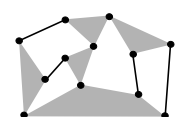

$9 / B_{7}$

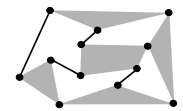

$9 / B_{14}$

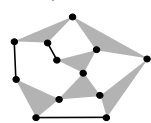

$9 / B_{21}$

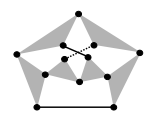

$9 / B_{28}$

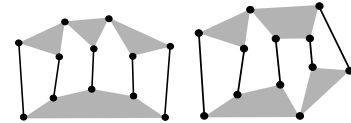

$9 / B_{1}$

$9 / B_{2}$

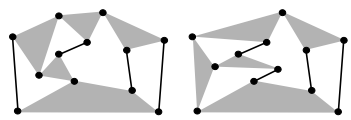

$9 / B_{8}$

$9 / B_{9}$
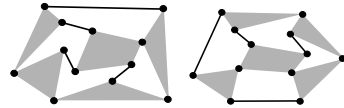

9/B 15

$9 / B_{16}$

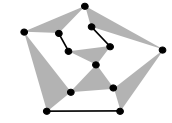

$9 / B_{22}$

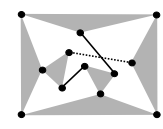

$9 / B_{23}$

Figure 1: The catalogued Baranov trusses.

equations is derived. This has been the dominating technique which, in general, requires not only complex eliminations but also tangent-half-angle substitutions. An even more compact formulation is obtained by a applying the following constructive process. Take one loop with a low number of joints and some of its joint variables as parameters which, when assigned to particular values, make the loop rigid. Then, the position of all links in the neighboring loops to this loop can also be obtained as a function of the chosen parameters taking, if needed, more joint variables as parameters. This process can be repeated till the locations of all links are expressed as functions of a set of parameters. Along the process, the locations of some links can be computed using different sets of parameters. This give rise to constraints between the parameters which translate into equations. The number of these equations is called the coupling degree of the truss [6, 12]. For non-overconstrained trusses, as is the case of Baranov trusses, the number of resulting constraints always equals the number of joint variables taken as parameters. Since the coupling degree is always lower than the number of loops, the elimination process to get a characteristic polynomial is simplified. Actually, when the coupling degree is 1 , eliminations are no longer required. Moreover, there are some trusses that form regular patterns whose coupling degree is independent from the number of its loops [11]. Although the idea is simple, its implementation using displacement transformations is not. This is probably why this approach has been belittled but, in this paper, we show how, by expressing the position analysis problem fully in terms of distances, this idea recovers interest because its implementation becomes straightforward. Moreover, as it will be presented in Section 4, for all the catalogued Baranov trusses the system of 
kinematic equations is reduced to a single scalar equation in one variable, except for the Baranov trusses $9 / B_{25}, 9 / B_{26}, 9 / B_{27}$, and $9 / B_{28}$ for which the system is formed by two scalar equations in two variables, a result in agreement with their coupling degrees found by Yang $[6]$.

This paper is organized as follows. Section 2 introduces the basic tools needed for the application of the proposed technique. Section 3 directly presents how the position analysis of the $9 / B_{28}$ Baranov truss can be solved using the presented tools. This is the most difficult case as it is one of the only four cases that requires an elimination process and, in addition, it has a characteristic polynomial of degree 58, the highest degree among all the catalogued Baranov trusses. Section 4 contains a discussion on the results obtained on the application of the proposed technique to all other catalogued Baranov trusses, which are summarized in Tables 2]11, and gives prospects for further research.

\section{Basic tools}

\subsection{Bilateration}

In what follows, $P_{i}$ will denote a point, $\overline{P_{i} P_{j}}$ the segment defined by $P_{i}$ and $P_{j}$, and $\triangle P_{i} P_{j} P_{k}$ the triangle defined by $P_{i}, P_{j}$, and $P_{k}$. Moreover, $\mathbf{p}_{i, j}=\overrightarrow{P_{i} P_{j}}$ and $s_{i, j}=$ $\left\|\mathbf{p}_{i, j}\right\|^{2}$.
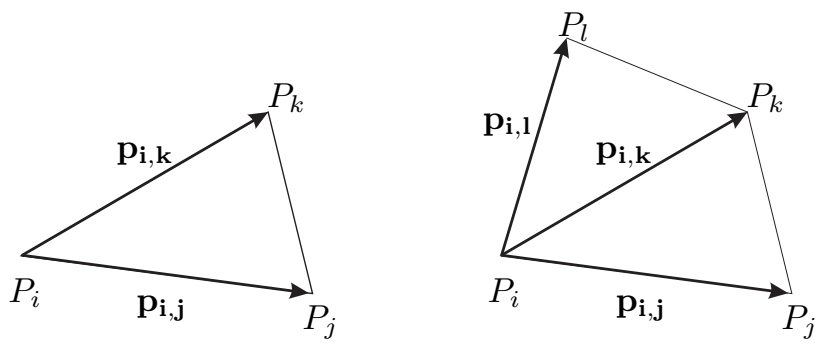

Figure 2: Left: The bilateration problem. Right: Concatenation of two bilaterations.

The bilateration problem consists in finding the feasible locations of a point, say $P_{k}$, given its distances to two other points, say $P_{i}$ and $P_{j}$, whose locations are known. Then, according to Fig. 2(left), the result to this problem, in matrix form, can be expressed as:

$$
\mathbf{p}_{i, k}=\mathbf{Z}_{i, j, k} \mathbf{p}_{i, j}
$$

where

$$
\mathbf{Z}_{i, j, k}=\frac{1}{2 s_{i, j}}\left[\begin{array}{cc}
s_{i, j}+s_{i, k}-s_{j, k} & -4 A_{i, j, k} \\
4 A_{i, j, k} & s_{i, j}+s_{i, k}-s_{j, k}
\end{array}\right]
$$

is called a bilateration matrix, and

$$
A_{i, j, k}= \pm \frac{1}{4} \sqrt{\left(s_{i, j}+s_{i, k}+s_{j, k}\right)^{2}-2\left(s_{i, j}^{2}+s_{i, k}{ }^{2}+s_{j, k}^{2}\right)}
$$

is the oriented area of $\triangle P_{i} P_{j} P_{k}$ which is defined as positive if $P_{k}$ is to the left of vector $\mathbf{p}_{i, j}$, and negative otherwise. The interested reader is addressed to [13] for a derivation of equation (11). 
Given the triangle in Fig. 2(left), it is possible to compute six different bilaterations. By algebraically manipulating the obtained results, it is possible to prove that:

$$
\begin{aligned}
& \mathbf{Z}_{i, j, k}=\mathbf{I}-\mathbf{Z}_{j, i, k}, \\
& \mathbf{Z}_{i, j, k}=\left(\mathbf{Z}_{i, k, j}\right)^{-1}, \\
& \mathbf{Z}_{i, j, k}=-\mathbf{Z}_{k, j, i} \mathbf{Z}_{j, i, k} .
\end{aligned}
$$

Moreover, it can be observed that the product of two bilateration matrices is commutative. Then, it is easy to prove that the set of bilateration matrices, that is, matrices of the form $\left(\begin{array}{cc}a & -b \\ b & a\end{array}\right)$, constitute a commutative group under the product and addition operations.

Another important property, that will be useful later, comes from the fact that if $\mathbf{v}=\mathbf{Z} \mathbf{w}$, where $\mathbf{Z}$ is a bilateration matrix, then $\|\mathbf{v}\|^{2}=\operatorname{det}(\mathbf{Z})\|\mathbf{w}\|^{2}$.

Now, let us consider the two triangles sharing one edge depicted in Fig. 2(right). Then, $\mathbf{p}_{j, l}$ can be expressed in terms of $\mathbf{p}_{j, l}$ by applying two consecutive bilaterations, as

$$
\mathbf{p}_{i, l}=\mathbf{Z}_{i, k, l} \mathbf{p}_{i, k}=\mathbf{Z}_{i, k, l} \mathbf{Z}_{i, j, k} \mathbf{p}_{i, j}
$$

Actually, any vector involving $P_{i}, P_{j}, P_{k}, P_{l}$ can be expressed in function of $\mathbf{p}_{i, j}$ using bilateration matrices. For example,

$$
\mathbf{p}_{j, l}=\mathbf{p}_{i, l}-\mathbf{p}_{i, j}=\left(\mathbf{Z}_{i, k, l} \mathbf{Z}_{i, j, k}-\mathbf{I}\right) \mathbf{p}_{i, j}
$$

As a consequence, the unknown squared distance between $P_{j}$ and $P_{l}$ can be obtained as:

$$
s_{j, l}=\operatorname{det}\left(\mathbf{Z}_{i, k, l} \mathbf{Z}_{i, j, k}-\mathbf{I}\right) s_{i, j} .
$$

If this result is compared to the one presented for example in [14, pp. 65-69], one starts to appreciate the ability of bilateration matrices to represent the solution of complex problems in a very compact form.

\subsection{Squared distances in strips of triangles}

The results presented in the previous subsection can be extended to strips of triangles -i.e., series of connected triangles that share one edge with one neighbor and another with the next. As an example, let us suppose that we are interested in finding $\mathbf{p}_{5,7}$ as a function of $\mathbf{p}_{1,2}$ for the strip of five triangles appearing in Fig. 3. Then, to this end, we can perform the following sequence of bilaterations:

$$
\begin{aligned}
\mathbf{p}_{5,7} & =\mathbf{Z}_{5,6,7} \mathbf{p}_{5,6} \\
& =\mathbf{Z}_{5,6,7} \mathbf{Z}_{5,4,7} \mathbf{p}_{5,4} \\
& =\mathbf{Z}_{5,6,7} \mathbf{Z}_{5,4,7}\left(\mathbf{p}_{1,4}+\mathbf{p}_{5,1}\right) \\
& =\mathbf{Z}_{5,6,7} \mathbf{Z}_{5,4,7}\left(\mathbf{I}-\mathbf{Z}_{1,4,5}\right) \mathbf{p}_{1,4} \\
& =\mathbf{Z}_{5,6,7} \mathbf{Z}_{5,4,7} \mathbf{Z}_{4,1,5} \mathbf{p}_{1,4} \\
& =\mathbf{Z}_{5,6,7} \mathbf{Z}_{5,4,7} \mathbf{Z}_{4,1,5} \mathbf{Z}_{1,3,4} \mathbf{p}_{1,3} \\
& =\mathbf{Z}_{5,6,7} \mathbf{Z}_{5,4,7} \mathbf{Z}_{4,1,5} \mathbf{Z}_{1,3,4} \mathbf{Z}_{1,2,3} \mathbf{p}_{1,2}
\end{aligned}
$$




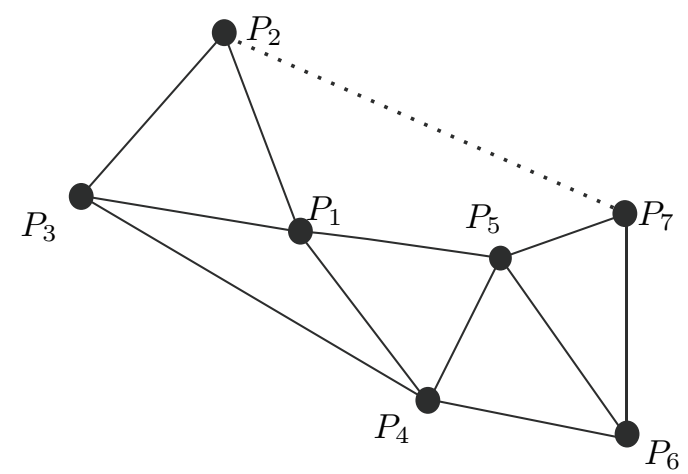

Figure 3: In a strip of triangles any unknown distance between any two of its vertices can be obtained by a set of bilaterations (see text for details).

Now, let us suppose that we want to compute $\mathbf{p}_{2,7}$ as a function of $\mathbf{p}_{1,2}$. In this case $\overline{P_{2} P_{7}}$ is not an edge of any triangle but clearly

$$
\begin{aligned}
\mathbf{p}_{2,7} & =-\mathbf{p}_{1,2}+\mathbf{p}_{1,5}+\mathbf{p}_{5,7} \\
& =-\mathbf{p}_{1,2}+\mathbf{p}_{1,5}+\mathbf{Z}_{5,6,7} \mathbf{Z}_{5,4,6} \mathbf{p}_{5,4} \\
& =-\mathbf{p}_{1,2}+\mathbf{p}_{1,5}+\mathbf{Z}_{5,6,7} \mathbf{Z}_{5,4,6}\left(\mathbf{p}_{1,4}+\mathbf{p}_{5,1}\right) \\
& =-\mathbf{p}_{1,2}+\mathbf{p}_{1,5}+\mathbf{Z}_{5,6,7} \mathbf{Z}_{5,4,6} \mathbf{Z}_{4,1,5} \mathbf{p}_{1,4} \\
& =-\mathbf{p}_{1,2}+\left(\mathbf{Z}_{1,4,5}+\mathbf{Z}_{5,6,7} \mathbf{Z}_{5,4,6} \mathbf{Z}_{4,1,5}\right) \mathbf{p}_{1,4} \\
& =\left(\left(\mathbf{Z}_{1,4,5}+\mathbf{Z}_{5,6,7} \mathbf{Z}_{5,4,6} \mathbf{Z}_{4,1,5}\right) \mathbf{Z}_{1,3,4} \mathbf{Z}_{1,2,3}-\mathbf{I}\right) \mathbf{p}_{1,2} .
\end{aligned}
$$

Therefore, the squared distance $s_{2,7}$ can be expressed, as a function of all other known distance in the strip, as:

$$
s_{2,7}=\operatorname{det}\left(\left(\mathbf{Z}_{1,4,5}+\mathbf{Z}_{5,6,7} \mathbf{Z}_{5,4,6} \mathbf{Z}_{4,1,5}\right) \mathbf{Z}_{1,3,4} \mathbf{Z}_{1,2,3}-\mathbf{I}\right) s_{1,2} .
$$

Observe that, if $s_{2,7}$ is fixed to a given value, the above equation can be seen as a closure equation, a condition that is fulfilled if and only if the strip of triangles can be assembled so that the distance between $P_{2}$ and $P_{7}$ is the desired one. This way of obtaining closure conditions is extended in the next subsection to complex trusses.

\subsection{Closure conditions using bilateration}

Fig. 4(a) shows a strip of triangles equivalent to a planar truss consisting of 9 binary links that conforms 4 non-oriented triangles. In this truss, once points $P_{1}$ and $P_{4}$ have been located on the plane, points $P_{2}$ and $P_{5}$ can be positioned in 8 and 4 different locations, respectively. If $\mathbf{p}_{1,6}$ is taken as reference, using a sequence of bilaterations, we have that

$$
s_{2,5}=\operatorname{det}\left(\mathbf{I}-\mathbf{Z}_{1,3,2} \mathbf{Z}_{1,6,3}-\mathbf{Z}_{6,4,5} \mathbf{Z}_{6,1,4}\right) s_{1,6} .
$$

Now, if the binary link $P_{1} P_{6}$ is removed, the planar truss becomes a four-bar linkage [Fig. 4(b)]. But, if a binary link is then added between $P_{2}$ and $P_{5}$, a truss is again 


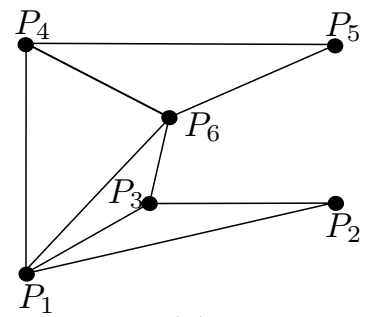

(a)

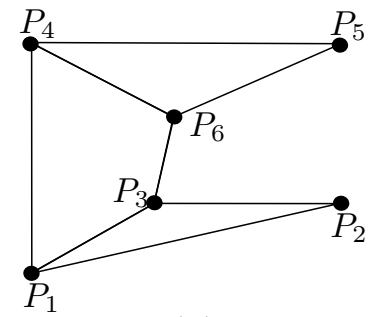

(b)

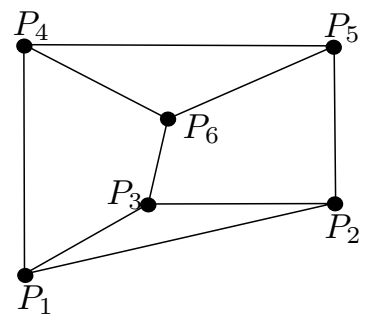

(c)

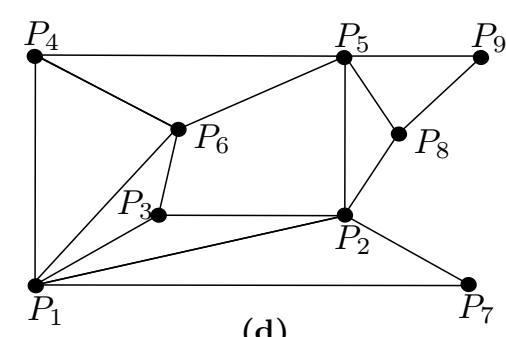

(d)

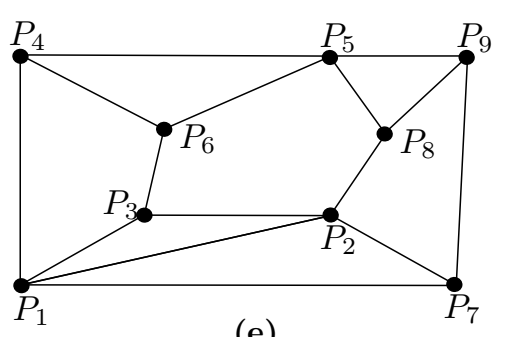

(e)

Figure 4: If the binary link $P_{1} P_{6}$ is removed in the strip of triangles appearing in (a), a four-bar linkage is obtained (b). If a binary link is then added between $P_{2}$ and $P_{5}$, a truss is again obtained (c). The strips of triangles $\left\{\triangle P_{1} P_{2} P_{7}, \triangle P_{1} P_{3} P_{2}, \triangle P_{1} P_{6} P_{3}, \triangle P_{1} P_{4} P_{6}, \triangle P_{4} P_{5} P_{6}\right\}$ and $\left\{\triangle P_{2} P_{5} P_{8}, \triangle P_{5} P_{9} P_{8}\right\}$ define a planar truss (d). If the binary links $P_{1} P_{6}$ and $P_{2} P_{5}$ are removed and a binary link is added between $P_{7}$ and $P_{9}$ a new truss is obtained whose closure condition can be expressed as the squared distance $s_{7,9}$ as a function of $s_{1,6}(\mathbf{e})$.

obtained [Fig. 4(c)]. What are the assembly modes of this new truss? Observe that the closure condition of this truss is given by equation (9). The solution of this equation gives the set of values of $s_{1,6}$ compatible with the lengths of all the binary links of the new truss. Actually, when $\triangle P_{1} P_{3} P_{2}$ and $\triangle P_{4} P_{5} P_{6}$ are oriented -i.e., when $\triangle P_{1} P_{3} P_{2}$ and $\triangle P_{4} P_{5} P_{6}$ are ternary links - this truss corresponds to the $5 / B_{1}$ Baranov truss.

The above process can be iterated further. Considering now that a new triangle is added to the strip of triangles of Fig. 4 (a) to the edge $\overline{P_{1} P_{2}}$, and that points $P_{2}$ and $P_{5}$

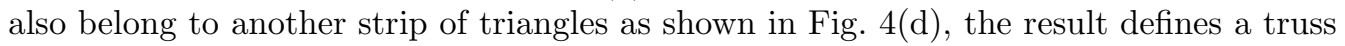
in which the squared distance $s_{7,9}$ can be easily determined. Then, if the binary links $\overline{P_{1} P_{6}}$ and $\overline{P_{2} P_{5}}$ are removed and a binary link is added between $P_{7}$ and $P_{9}$ a new truss is obtained [Fig. [(e)]. The assembly modes of this new truss can be computed using the expression obtained for $s_{7,9}$, a scalar equation in $s_{1,6}$. Actually, when $\triangle P_{1} P_{3} P_{2}$, $\triangle P_{1} P_{2} P_{7}, \triangle P_{4} P_{5} P_{6}$, and $\triangle P_{5} P_{9} P_{8}$ are oriented, the resulting truss corresponds to the $7 / B_{3}$ Baranov truss.

\subsection{Closure conditions and symmetries}

The symmetries of a truss are given by its automorphisms. An automorphism of a truss is a set of permutations of its joints that map the truss onto itself while preserving the connectivity between joints. The composition of two automorphisms is clearly another automorphism, and the set of automorphisms of a given truss, under the composition operation, forms a group, the automorphism group of the truss.

Finding the automorphism group and an irreducible set of its generators for a Baranov truss is an easy task using any of the available open-source software tools for computing 


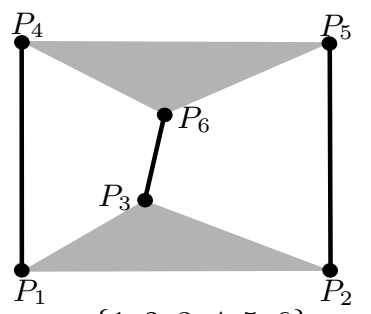

$\{1,2,3,4,5,6\}$

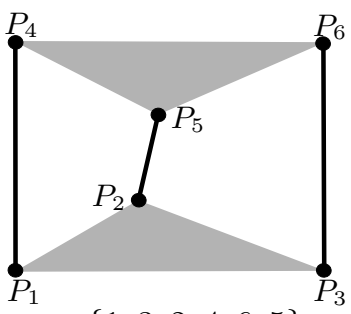

$\{1,3,2,4,6,5\}$

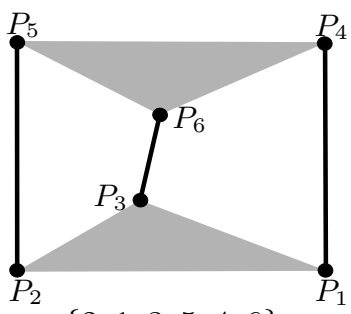

$\{2,1,3,5,4,6\}$

(a)

(b)

Figure 5: Three of the twelve automorphisms of the $5 / B_{1}$ Baranov truss.

graph automorphisms. For instance, the $5 / B_{1}$ Baranov truss, according to the notation of Fig. 过(c), has twelve automorphisms: $\{1,2,3,4,5,6\},\{1,3,2,4,6,5\},\{2,1,3,5,4,6\}$, $\{2,3,1,5,6,4\},\{3,1,2,6,4,5\},\{3,2,1,6,5,4\},\{4,5,6,1,2,3\},\{4,6,5,1,3,2\},\{5,4,6$, $2,1,3\},\{5,6,4,2,3,1\},\{6,4,5,3,1,2\}$, and $\{6,5,4,3,2,1\}$ (Fig. [5 depicts the first three). Observe that the closure condition of any of these symmetric trusses is obtained by the simple application of the corresponding permutation to equation (9). For example, the closure condition of the truss in Fig. F(b) is obtained by applying the permutation

$$
\left[\begin{array}{llllll}
1 & 2 & 3 & 4 & 5 & 6 \\
1 & 3 & 2 & 4 & 6 & 5
\end{array}\right]
$$

That is,

$$
s_{3,6}=\operatorname{det}\left(\mathbf{I}-\mathbf{Z}_{1,2,3} \mathbf{Z}_{1,5,2}-\mathbf{Z}_{5,4,6} \mathbf{Z}_{5,1,4}\right) s_{1,5} .
$$

\section{Example: The $9 / B_{28}$ Baranov truss}

The $9 / B_{28}$ Baranov truss is one of the three catalogued Baranov trusses that cannot be represented as a planar graph (the other two are $9 / B_{23}$ and $\left.9 / B_{24}\right)$ [6], [15]. It was characterized for the first time by Baranov in 1952 [4] and Wang et. al. developed a procedure based on a complex number method and Dixon resultant to solve its position analysis in closed form [16]. They obtained a univariate polynomial of degree 64 but 6 extraneous roots were found, leading to the conclusion it can have up to 58 assembly modes - the largest number of assembly modes for a catalogued Baranov truss - , a result in agreement with that obtained by Hang et. al. using homotopy continuation [8].

In the $9 / B_{28}$ Baranov truss depicted in Fig. 6 6 (a), the revolute pair centers of the six ternary links define the triangles $\triangle P_{1} P_{8} P_{3}, \triangle P_{3} P_{6} P_{4}, \triangle P_{4} P_{7} P_{5}, \triangle P_{2} P_{5} P_{11}, \triangle P_{8} P_{12} P_{9}$, and $\triangle P_{10} P_{12} P_{11}$. Next, it is shown how the kinematic equations of this truss can be reduced to compute $s_{6,10}$ and $s_{7,9}$ as a function of $s_{1,4}$ and $s_{2,4}$. That is, $s_{1,4}$ and $s_{2,4}$ are used as parameters in terms of which the location of all other links of the truss can be expressed. Since two parameters are needed, the truss is said to have coupling number 2 , as already observed by $[6]$. 


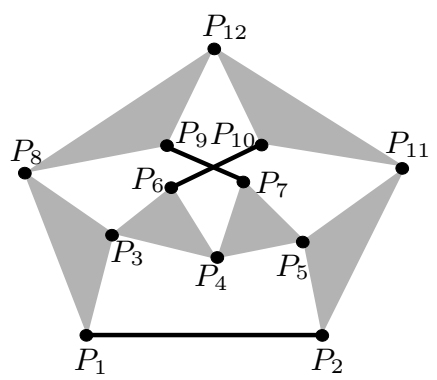

(a)

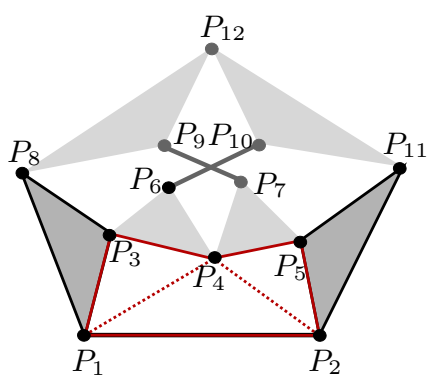

(b)

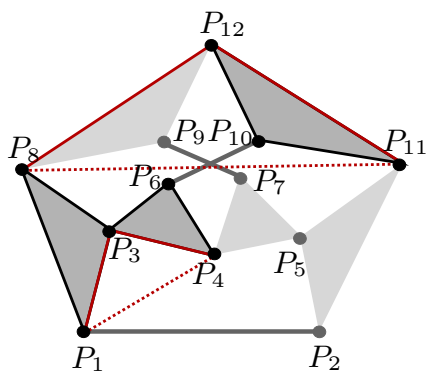

(c)

Figure 6: A 9/B $B_{28}$ Baranov truss (a). The strip of triangles $\left\{\triangle P_{1} P_{8} P_{3}, \triangle P_{1} P_{3} P_{4}, \triangle P_{1} P_{4} P_{2}, \triangle P_{4} P_{2} P_{5}\right.$, $\left.\triangle P_{2} P_{5} P_{11}\right\}$ has been considered to compute $s_{8,11}$ as a function of $s_{1,4}$ and $s_{2,4}$ (b). The strips of triangles $\left\{\triangle P_{8} P_{12} P_{11}, \triangle P_{10} P_{12} P_{11}\right\},\left\{\triangle P_{1} P_{8} P_{3}, \triangle P_{1} P_{3} P_{4}\right\}$, and $\left\{\triangle P_{3} P_{4} P_{6}, \triangle P_{1} P_{3} P_{4}\right\}$ have also been considered to compute $s_{6,10}$ as a function of $s_{1,4}$ (c).

\subsection{Computing $s_{6,10}$ as a function of $s_{1,4}$ and $s_{2,4}$}

On the one hand, for the strip of triangles in Fig. 6(b), we have

$$
\begin{aligned}
\mathbf{p}_{8,11} & =-\mathbf{p}_{1,8}+\mathbf{p}_{1,4}-\mathbf{p}_{2,4}+\mathbf{p}_{2,11} \\
& =\left(-\mathbf{Z}_{1,3,8} \mathbf{Z}_{1,4,3}+\mathbf{I}\right) \mathbf{p}_{1,4}+\left(\mathbf{Z}_{2,5,11} \mathbf{Z}_{2,4,5}-\mathbf{I}\right) \mathbf{p}_{2,4} \\
& =\left(-\mathbf{Z}_{1,3,8} \mathbf{Z}_{1,4,3}+\mathbf{I}+\left(\mathbf{Z}_{2,5,11} \mathbf{Z}_{2,4,5}-\mathbf{I}\right) \mathbf{Z}_{4,1,2}\right) \mathbf{p}_{1,4}
\end{aligned}
$$

Therefore,

$$
s_{8,11}=f\left(s_{1,4}, s_{2,4}\right)=\operatorname{det}\left(-\mathbf{Z}_{1,3,8} \mathbf{Z}_{1,4,3}+\mathbf{I}-\mathbf{Z}_{4,1,2}+\mathbf{Z}_{2,5,11} \mathbf{Z}_{2,4,5} \mathbf{Z}_{4,1,2}\right) s_{1,4} .
$$

On the other hand, from the three strips of triangles in Fig. 6)(c),

$$
\begin{aligned}
\mathbf{p}_{6,10} & =-\mathbf{p}_{1,6}+\mathbf{p}_{1,8}+\mathbf{p}_{8,10} \\
& =\left(\mathbf{Z}_{4,3,6} \mathbf{Z}_{4,1,3}-\mathbf{I}\right) \mathbf{p}_{1,4}+\mathbf{Z}_{1,3,8} \mathbf{Z}_{1,4,3} \mathbf{p}_{1,4}+\left(\mathbf{I}-\mathbf{Z}_{11,12,10} \mathbf{Z}_{11,8,12}\right) \mathbf{p}_{8,11} .
\end{aligned}
$$

Then, the substitution of equation (10) in equation (12) yields

$$
\begin{aligned}
\mathbf{p}_{6,10}= & \left(-\mathbf{I}+\mathbf{Z}_{4,3,6} \mathbf{Z}_{4,1,3}+\mathbf{Z}_{1,3,8} \mathbf{Z}_{1,4,3}+\left(\mathbf{I}-\mathbf{Z}_{11,12,10} \mathbf{Z}_{11,8,12}\right)\left(-\mathbf{Z}_{1,3,8} \mathbf{Z}_{1,4,3}\right.\right. \\
& \left.\left.+\mathbf{I}-\mathbf{Z}_{4,1,2}+\mathbf{Z}_{2,5,11} \mathbf{Z}_{2,4,5} \mathbf{Z}_{4,1,2}\right)\right) \mathbf{p}_{1,4} .
\end{aligned}
$$

Therefore,

$$
\begin{aligned}
s_{6,10}= & f\left(s_{1,4}, s_{2,4}, s_{8,11}\right) \\
= & \operatorname{det}\left(-\mathbf{I}+\mathbf{Z}_{4,3,6} \mathbf{Z}_{4,1,3}+\mathbf{Z}_{1,3,8} \mathbf{Z}_{1,4,3}+\left(\mathbf{I}-\mathbf{Z}_{11,12,10} \mathbf{Z}_{11,8,12}\right)\left(-\mathbf{Z}_{1,3,8} \mathbf{Z}_{1,4,3}\right.\right. \\
& \left.\left.+\mathbf{I}-\mathbf{Z}_{4,1,2}+\mathbf{Z}_{2,5,11} \mathbf{Z}_{2,4,5} \mathbf{Z}_{4,1,2}\right)\right) s_{1,4}
\end{aligned}
$$

Then, the substitution of equation (11) in equation (14) yields a scalar equation in two variables: $s_{1,4}$ and $s_{2,4}$. 


\subsection{Computing $s_{7,9}$ as a function of $s_{1,4}$ and $s_{2,4}$}

The computation of $s_{7,9}$ as a function of $s_{2,4}$ and $s_{2,4}$ can be simplified by considering the symmetries of the analyzed truss. Indeed, by applying the permutation

$$
\left[\begin{array}{cccccccccccc}
1 & 2 & 3 & 4 & 5 & 6 & 7 & 8 & 9 & 10 & 11 & 12 \\
2 & 1 & 5 & 4 & 3 & 7 & 6 & 11 & 10 & 9 & 8 & 12
\end{array}\right]
$$

to equation (14), we conclude that

$$
\begin{aligned}
s_{7,9}= & f\left(s_{1,4}, s_{2,4}, s_{8,11}\right) \\
= & \operatorname{det}\left(-\mathbf{I}+\mathbf{Z}_{4,5,7} \mathbf{Z}_{4,2,5}+\mathbf{Z}_{2,5,11} \mathbf{Z}_{2,4,5}+\left(\mathbf{I}-\mathbf{Z}_{8,12,9} \mathbf{Z}_{8,11,12}\right)\left(-\mathbf{Z}_{2,5,11} \mathbf{Z}_{2,4,5}\right.\right. \\
& \left.\left.+\mathbf{I}-\mathbf{Z}_{4,2,1}+\mathbf{Z}_{1,3,8} \mathbf{Z}_{1,4,3} \mathbf{Z}_{4,2,1}\right)\right) s_{2,4} .
\end{aligned}
$$

The expansion of the right hand side of equations (14) and (15), using equation (11) for substituting the unknown squared distance $s_{1,8}$, yields a system of two scalar equations in two variables: $s_{1,4}$ and $s_{2,4}$. Next, it is shown how to algebraically manipulate this system to obtain the characteristic polynomial of the $9 / B_{28}$ Baranov truss.

\subsection{Deriving the characteristic polynomial}

The characteristic polynomial of the $9 / B_{28}$ Baranov truss can be obtained by: (i) converting the scalar radical equations (14) and (15) in polynomials equations in $s_{1,4}$ and $s_{2,4}$, say $\mathcal{P}_{1}\left(s_{1,4}, s_{2,4}\right)=0$ and $\mathcal{P}_{2}\left(s_{1,4}, s_{2,4}\right)=0$, respectively, by clearing all the involved square roots; and (ii) eliminating $s_{2,4}$, or $s_{1,4}$, from the resulting polynomial system to get a single polynomial in one variable.

By expanding equation (11), we get

$$
\begin{aligned}
s_{8,11}= & \frac{1}{s_{1,4} s_{2,4}}\left(\Lambda_{1}+\Lambda_{2} A_{1,4,3}+\Lambda_{3} A_{1,4,2}+\Lambda_{4} A_{2,4,5}+\Lambda_{5} A_{1,4,3} A_{1,4,2}+\Lambda_{6} A_{1,4,3}\right. \\
& \left.A_{2,4,5}+\Lambda_{7} A_{1,4,2} A_{2,4,5}+\Lambda_{8} A_{1,4,3} A_{1,4,2} A_{2,4,5}\right)
\end{aligned}
$$

where $A_{1,4,3}, A_{1,4,2}$, and $A_{2,4,5}$ are the unknown oriented areas of $\triangle P_{1} P_{4} P_{3}, \triangle P_{1} P_{4} P_{2}$, and $\triangle P_{2} P_{4} P_{5}$, respectively, and $\Lambda_{i}, i=1, \ldots, 8$ are polynomials in $s_{1,4}$ and $s_{2,4}$.

Likewise, by expanding equation (14), we get

$$
s_{6,10}=\frac{1}{s_{1,4} s_{2,4} s_{8,11}} \Psi
$$

where

$$
\begin{aligned}
\Psi= & \Psi_{1}+\Psi_{2} A_{1,4,3}+\Psi_{3} A_{1,4,2}+\Psi_{4} A_{2,4,5}+\Psi_{5} A_{8,11,12}+\Psi_{6} A_{1,4,3} A_{1,4,2}+\Psi_{7} A_{1,4,3} \\
& A_{2,4,5}+\Psi_{8} A_{1,4,3} A_{8,11,12}+\Psi_{9} A_{1,4,2} A_{2,4,5}+\Psi_{10} A_{1,4,2} A_{8,11,12}+\Psi_{11} A_{2,4,5} \\
& A_{8,11,12}+\Psi_{12} A_{1,4,3} A_{1,4,2} A_{2,4,5}+\Psi_{13} A_{1,4,3} A_{1,4,2} A_{8,11,12}+\Psi_{14} A_{1,4,3} A_{2,4,5} \\
& A_{8,11,12}+\Psi_{15} A_{1,4,2} A_{2,4,5} A_{8,11,12}+\Psi_{16} A_{1,4,3} A_{1,4,2} A_{2,4,5} A_{8,11,12}
\end{aligned}
$$


with $\Psi_{i}, i=1, \ldots, 16$, polynomials in $s_{1,4}, s_{2,4}$ and $s_{8,11}$.

Now, by expressing equation (17) as a linear equation in $A_{8,11,12}$ (i.e., $a+b A_{8,11,12}$ $=0$ ), squaring it (i.e., $a^{2}-b^{2} A_{8,11,12}^{2}=0$ ), and replacing equation (16) in the result, a equation in $A_{1,4,3}, A_{1,4,2}$, and $A_{2,4,5}$ is obtained. Repeating this process for $A_{2,4,5}$, we get

$$
\Phi_{1}+\Phi_{2} A_{1,4,3}+\Phi_{3} A_{1,4,2}+\Phi_{4} A_{1,4,3} A_{1,4,2}=0,
$$

where $\Phi_{1}, \Phi_{2}, \Phi_{3}$, and $\Phi_{4}$ are polynomials in $s_{1,4}$ of degree $16,15,15$, and 14 , respectively, and in $s_{2,4}$ of degree 8 in all cases. Then, to clear radicals, the terms in equation (18) can be rearranged to obtain

$$
\Phi_{2} A_{1,4,3}+\Phi_{3} A_{1,4,2}=-\left(\Phi_{1}+\Phi_{4} A_{1,4,3} A_{1,4,2}\right) .
$$

Now, by squaring both sides of the above equation, expanding the result, and rearranging terms, we get

$$
\Phi_{2}^{2} A_{1,4,3}^{2}+\Phi_{3}^{2} A_{1,4,2}^{2}-\Phi_{4}^{2} A_{1,4,3}^{2} A_{1,4,2}^{2}-\Phi_{1}^{2}=2\left(\Phi_{1} \Phi_{4}-\Phi_{2} \Phi_{3}\right) A_{1,4,3} A_{1,4,2} .
$$

Finally, if both sides of the above equation are again squared and expanded, the following equation is obtained:

$$
\begin{aligned}
& -\Phi_{4}^{4} A_{1,4,3}^{4} A_{1,4,2}^{4}+2 \Phi_{4}^{2} \Phi_{2}^{2} A_{1,4,3}^{4} A_{1,4,2}^{2}+2 \Phi_{4}^{2} \Phi_{3}^{2} A_{1,4,3}^{2} A_{1,4,2}^{4}-\Phi_{2}^{4} A_{1,4,3}^{4}-\Phi_{3}^{4} A_{1,4,2}^{4} \\
& -\Phi_{1}^{4}+\left(2 \Phi_{2}^{2} \Phi_{3}^{2}-8 \Phi_{2} \Phi_{3} \Phi_{4} \Phi_{1}+2 \Phi_{4}^{2} \Phi_{1}^{2}\right) A_{1,4,3}^{2} A_{1,4,2}^{2}+2 \Phi_{1}^{2} \Phi_{2}^{2} A_{1,4,3}^{2}+2 \Phi_{1}^{2} \Phi_{3}^{2} A_{1,4,2}^{2}=0 .
\end{aligned}
$$

If the above procedure is applied to equation (16), we get a polynomial in $s_{1,4}, s_{2,4}$, and $s_{8,11}$, say $\mathcal{F}\left(s_{1,4}, s_{2,4}, s_{8,11}\right)$. Finally, the full expansion of equation (19) factorizes as:

$$
s_{1,4}^{16} s_{2,4}^{16} \mathcal{F}\left(s_{1,4}, s_{2,4}, 0\right) \mathcal{P}_{1}=0
$$

where $\mathcal{P}_{1}$ is a non-homogeneous bivariate polynomial in $s_{1,4}$ and $s_{2,4}$ with leading term $s_{1,4}^{32} s_{2,4}^{14}$. The roots of the term $s_{1,4}^{16} s_{2,4}^{16} \mathcal{F}\left(s_{1,4}, s_{2,4}, 0\right)$ were introduced when clearing denominators to obtain equation (17), so they can be dropped. To obtain $\mathcal{P}_{2}$, we can proceed in the same way as for the derivation of $\mathcal{P}_{1}$.

Finally, to obtain the characteristic polynomial, we have to eliminate either $s_{2,4}$ or $s_{1,4}$ from the polynomial system $\left\{\mathcal{P}_{1}\left(s_{1,4}, s_{2,4}\right)=0, \mathcal{P}_{2}\left(s_{1,4}, s_{2,4}\right)=0\right\}$. This can be implemented using, for example, Sylvester or Bézout resultants. If we eliminate either $s_{2,4}$ or $s_{1,4}$, the associated Sylvester and Bézout matrices have dimensions $68 \times 68$ and $36 \times 36$, respectively. In any case - i.e., eliminating either $s_{2,4}$ or $s_{1,4}$ and using either Sylvester or the Bézout resultants - the result is a polynomial equation of degree 1826 . When $s_{2,4}$ is the eliminated variable, this polynomial factors into 15 polynomials

$$
\mathcal{T}\left(s_{1,4}\right) \prod_{i=1}^{14} \mathcal{D}_{i}\left(s_{1,4}\right)=0
$$

where the roots of polynomials $\mathcal{D}_{1}, \ldots, \mathcal{D}_{14}$ are not solutions of the original system of radical equations formed by equations (14) and (15) and $\mathcal{T}$, the characteristic polynomial 
of the $9 / B_{28}$ Baranov truss, is of degree 58 in $s_{1,4}$. As expected, the same result is obtained when $s_{1,4}$ is the eliminated variable.

For each of the real roots of $\mathcal{T}\left(s_{1,4}\right)=0$, we can determine the Cartesian position of the revolute pair centers given by $P_{2}, P_{4}, P_{5}, P_{6}, P_{7}, P_{9}, P_{10}, P_{11}$, and $P_{12}$, with respect to the ternary link given by $\triangle P_{1} P_{8} P_{3}$, computing $s_{2,4}$ from the system $\left\{\mathcal{P}_{1}\left(s_{1,4}, s_{2,4}\right)=\right.$ $\left.0, \mathcal{P}_{2}\left(s_{1,4}, s_{2,4}\right)=0\right\}$ and using the set of equations

$$
\begin{aligned}
\mathbf{p}_{1,4} & =\mathbf{Z}_{1,3,4} \mathbf{p}_{1,3} \\
\mathbf{p}_{3,6} & =\mathbf{Z}_{3,4,6} \mathbf{p}_{3,4} \\
\mathbf{p}_{1,2} & =\mathbf{Z}_{1,4,2} \mathbf{p}_{1,4} \\
\mathbf{p}_{2,5} & =\mathbf{Z}_{2,4,5} \mathbf{p}_{2,4} \\
\mathbf{p}_{5,7} & =\mathbf{Z}_{4,5,7} \mathbf{p}_{4,5} \\
\mathbf{p}_{2,11} & =\mathbf{Z}_{2,5,11} \mathbf{p}_{2,5} \\
\mathbf{p}_{8,12} & =\mathbf{Z}_{8,11,12} \mathbf{p}_{8,11} \\
\mathbf{p}_{8,9} & =\mathbf{Z}_{8,12,9} \mathbf{p}_{8,12} \\
\mathbf{p}_{11,10} & =\mathbf{Z}_{11,12,10} \mathbf{p}_{11,12} .
\end{aligned}
$$

This process leads up to 16 combinations of locations for the couples $P_{6}, P_{10}$ and $P_{7}$, $P_{9}$, and at least one of them must satisfy simultaneously the distances imposed by the binary links connecting them.

\subsection{Numerical example}

According to the notation used in Fig. [6] let us suppose that $s_{1,2}=3185, s_{1,3}=\frac{6610}{9}$, $s_{1,8}=\frac{16525}{9}, s_{2,5}=\frac{820}{9}, s_{2,11}=\frac{15826}{9}, s_{3,4}=225, s_{3,6}=180, s_{3,8}=661, s_{4,5}=400$, $s_{4,6}=225, s_{4,7}=452, s_{5,7}=676, s_{5,11}=1202, s_{6,10}=625, s_{7,9}=625, s_{8,9}=305$, $s_{8,12}=1600, s_{9,12}=625, s_{10,11}=676, s_{10.12}=484$, and $s_{11,12}=1600$. This corresponds to the example used by Wang et. al. in [16]. Then, proceeding as explained above, we obtain the characteristic polynomial

$$
\begin{aligned}
& 1.848110^{239} s_{1,4}{ }^{58}-1.572510^{244} s_{1,4}{ }^{57}+6.585710^{248} s_{1,4}{ }^{56}-1.809910^{253} s_{1,4}{ }^{55} \\
& +3.671210^{257} s_{1,4}{ }^{54}-5.861010^{261} s_{1,4}{ }^{53}+7.669210^{265} s_{1,4}{ }^{52}-8.457110^{269} s_{1,4}{ }^{51} \\
& +8.019910^{273} s_{1,4}{ }^{50}-6.641310^{277} s_{1,4}{ }^{49}+4.860410^{281} s_{1,4}{ }^{48}-3.173710^{285} s_{1,4}{ }^{47} \\
& +1.863510^{289} s_{1,4}{ }^{46}-9.901910^{292} s_{1,4}{ }^{45}+4.787110^{296} s_{1,4}{ }^{44}-2.115210^{300} s_{1,4}{ }^{43} \\
& +8.573710^{303} s_{1,4}{ }^{42}-3.198510^{307} s_{1,4}{ }^{41}+1.101210^{311} s_{1,4}{ }^{40}-3.507310^{314} s_{1,4}{ }^{39} \\
& +1.035410^{318} s_{1,4}{ }^{38}-2.837910^{321} s_{1,4}{ }^{37}+7.232410^{324} s_{1,4}{ }^{36}-1.715810^{328} s_{1,4}{ }^{35} \\
& +3.793010^{331} s_{1,4}{ }^{34}-7.819410^{334} s_{1,4}{ }^{33}+1.504210^{338} s_{1,4}{ }^{32}-2.701210^{341} s_{1,4}{ }^{31} \\
& +4.529610^{344} s_{1,4}{ }^{30}-7.093710^{347} s_{1,4}{ }^{29}+1.037510^{351} s_{1,4}{ }^{28}-1.416810^{354} s_{1,4}{ }^{27} \\
& +1.806010^{357} s_{1,4}{ }^{26}-2.147910^{360} s_{1,4}{ }^{25}+2.381710^{363} s_{1,4}{ }^{24}-2.460310^{366} s_{1,4}{ }^{23} \\
& +2.365510^{369} s_{1,4}{ }^{22}-2.114110^{372} s_{1,4}{ }^{21}+1.754010^{375} s_{1,4}{ }^{20}-1.348710^{378} s_{1,4}{ }^{19} \\
& +9.593010^{380} s_{1,4}{ }^{18}-6.297910^{383} s_{1,4}{ }^{17}+3.806610^{386} s_{1,4}{ }^{16}-2.112110^{389} s_{1,4}{ }^{15}
\end{aligned}
$$



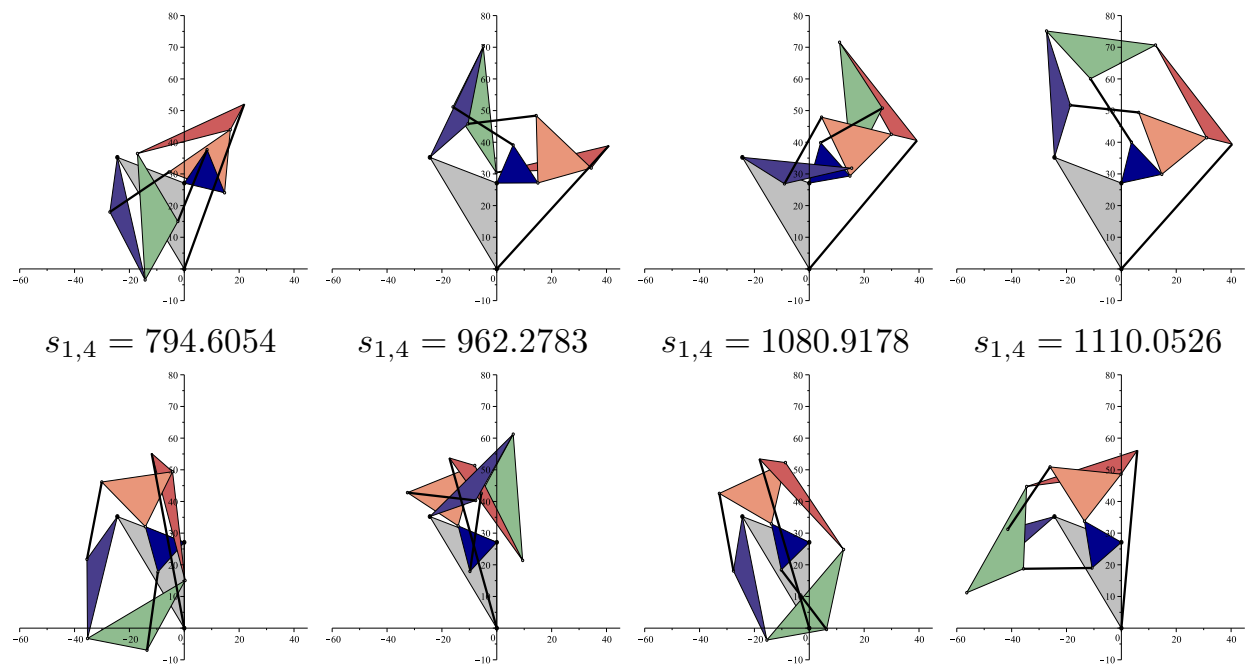

$s_{1,4}=1080.9178$

$s_{1,4}=1110.0526$
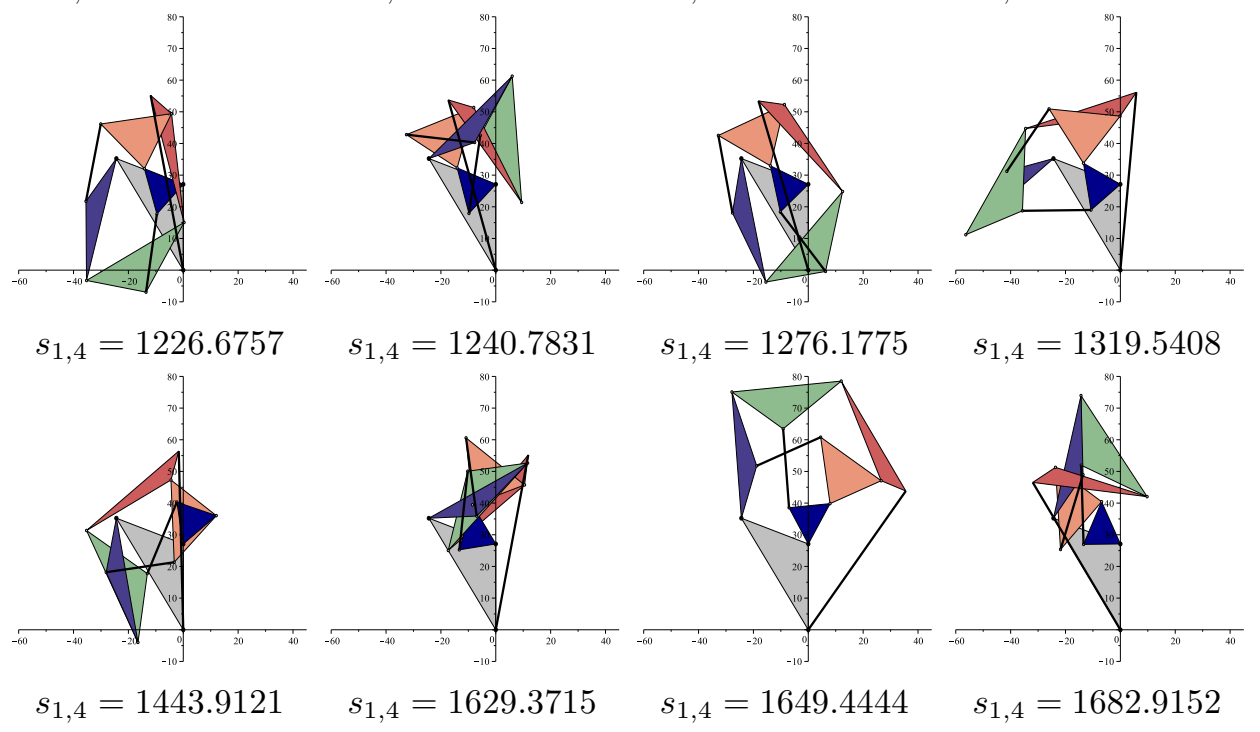

$s_{1,4}=1649.4444$

$s_{1,4}=1682.9152$
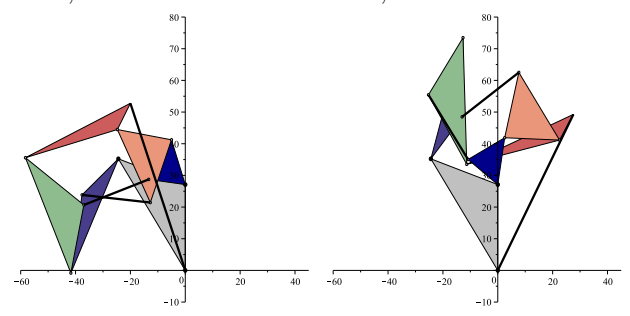

$s_{1,4}=1725.5533 \quad s_{1,4}=1760.9329$

Figure 7: The assembly modes of the analyzed 9/B28 Baranov truss. 


$$
\begin{aligned}
& +1.072210^{392} s_{1,4}{ }^{14}-4.959810^{394} s_{1,4}{ }^{13}+2.081210^{397} s_{1,4}{ }^{12}-7.878310^{399} s_{1,4}{ }^{11} \\
& +2.673110^{402} s_{1,4}{ }^{10}-8.066510^{404} s_{1,4}{ }^{9}+2.144210^{407} s_{1,4}{ }^{8}-4.961010^{409} s_{1,4}{ }^{7} \\
& +9.839510^{411} s_{1,4}{ }^{6}-1.639610^{414} s_{1,4}{ }^{5}+2.232710^{416} s_{1,4}{ }^{4}-2.386510^{418} s_{1,4}{ }^{3} \\
& +1.878010^{420} s_{1,4}{ }^{2}-9.676810^{421} s_{1,4}+2.449910^{423}
\end{aligned}
$$

This polynomial has 14 real roots, a result in agreement with the solution reported in [16]. The values of these roots, as well as the corresponding assembly modes, for the case in which $P_{1}=(0,0)^{T}, P_{3}=\left(0, \frac{1}{3} \sqrt{6610}\right)^{T}$, and $P_{8}=\left(-\frac{3}{10} \sqrt{6610}, \frac{13}{30} \sqrt{6610}\right)^{T}$, appear in Fig. 7. The coefficients of the above polynomial have to be computed in exact rational arithmetic. Otherwise, numerical problems make impracticable the correct computation of its roots. Although these coefficients are given here in floating point arithmetic for space limitation reasons, they could be of interest for comparison with other possible methods. To our knowledge, this is the first time that the characteristic polynomial of a $9 / B_{28}$ Baranov truss is explicitly obtained.

\section{Discussion and conclusions}

The simplest Baranov truss is the $3 / B_{1}$ truss or triad. This truss has three links and up to two assembly modes. Its position analysis is equivalent to solve the bilateration problem. Other analytical approaches can be found, for instance, in [17], [18]. When the technique described in this paper is applied to the position analysis of the other catalogued Baranov trusses, one observes that the problem is reduced to solve a single scalar radical equation in one variable for all cases, except for trusses $9 / B_{25}, 9 / B_{26}$, $9 / B_{27}$, and $9 / B_{28}$, for which the resulting system is formed by two scalar radical equations in two variables. This is in agreement with the coupling degree of the catalogued Baranov trusses presented in [6].

Since for the Baranov trusses with coupling degree 1 the obtained system of kinematic equations is a single scalar radical equation, their characteristic polynomials are obtained by simply clearing square roots, as it was already explained for trusses $7 / B_{1}, 7 / B_{2}$, and $7 / B_{3}$ in [13]. Obtaining the characteristic polynomials of trusses $9 / B_{25}, 9 / B_{26}$, $9 / B_{27}$, and $9 / B_{28}$ requires converting the resulting scalar radical equations in bivariate polynomials, by clearing square roots as well, and using classical elimination techniques, as it has been explained in this paper. Then, it is important to realize that 197 trusses, out of the total of 239 Baranov trusses with 5 loops, could also be solved in an elementary way and, for the remaining 42 trusses, the problem could be reduced to the solution of a system of two equations in two variables. Thus, compared to the approaches based on vector loop equations and elimination techniques, the application of the proposed technique to the position analysis of Baranov trusses seems clearly superior.

Tables 2 to 11 present, for all the catalogued Baranov trusses, the system of kinematic equations derived using the presented technique, the number of resulting assembly modes, and references to previous reported solutions using not only analytical approaches but also numerical ones. Using the kinematic equations presented in these tables, the closedform position analysis of trusses $9 / B_{2}, 9 / B_{3}, 9 / B_{4}, 9 / B_{5}, 9 / B_{6}, 9 / B_{8}, 9 / B_{9}, 9 / B_{13}$, $9 / B_{18}, 9 / B_{19}$, and $9 / B_{22}$, can be straightforwardly solved for the first time for the best of our knowledge. It can also be concluded from these tables that the number of assembly 


Baranov truss
$\mathbf{A M}$

Table 2: Position analysis of all the catalogued Baranov trusses (part 1).

modes previously reported in the literature for trusses $9 / B_{4}, 9 / B_{8}, 9 / B_{18}$, and $9 / B_{19}$ do not seem accurate.

Finally, it is worth to mention that the concept of Baranov truss has been extended to trusses with joints involving more than two links. It has been shown that there are such 125 Baranov trusses with up to four loops [19]. The closed-form solution to the position analysis of at least one of these trusses, the Dixon-Wunderlich linkage, has already been reported [20]. The application of the proposed technique to all other members of this family of trusses is a point that deserves further attention.

\section{Acknowledgements}

We gratefully acknowledge the financial support of the Colombian Ministry of Communications and Colfuturo through the ICT National Plan of Colombia. 


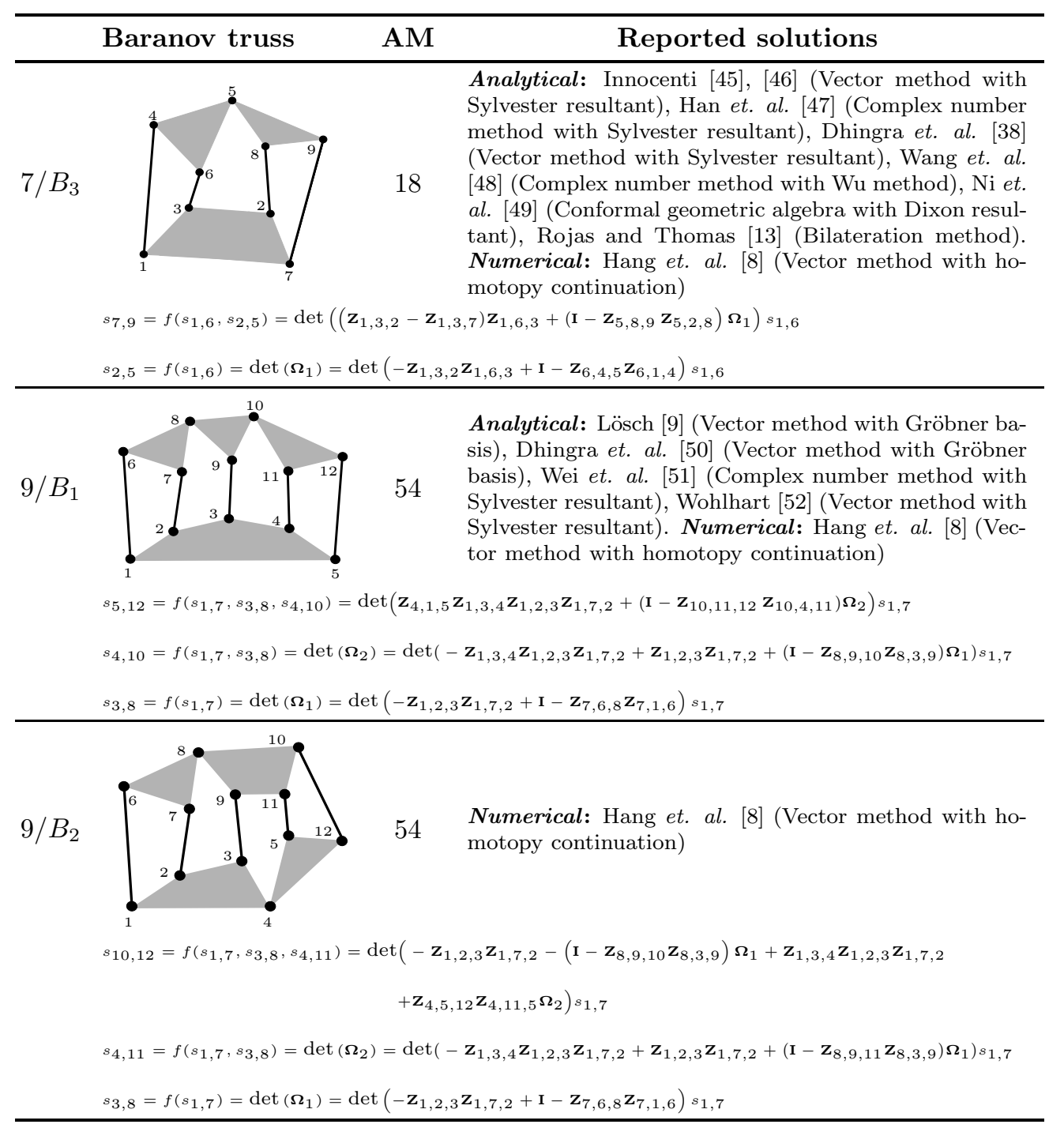

Table 3: Position analysis of all the catalogued Baranov trusses (part 2). 


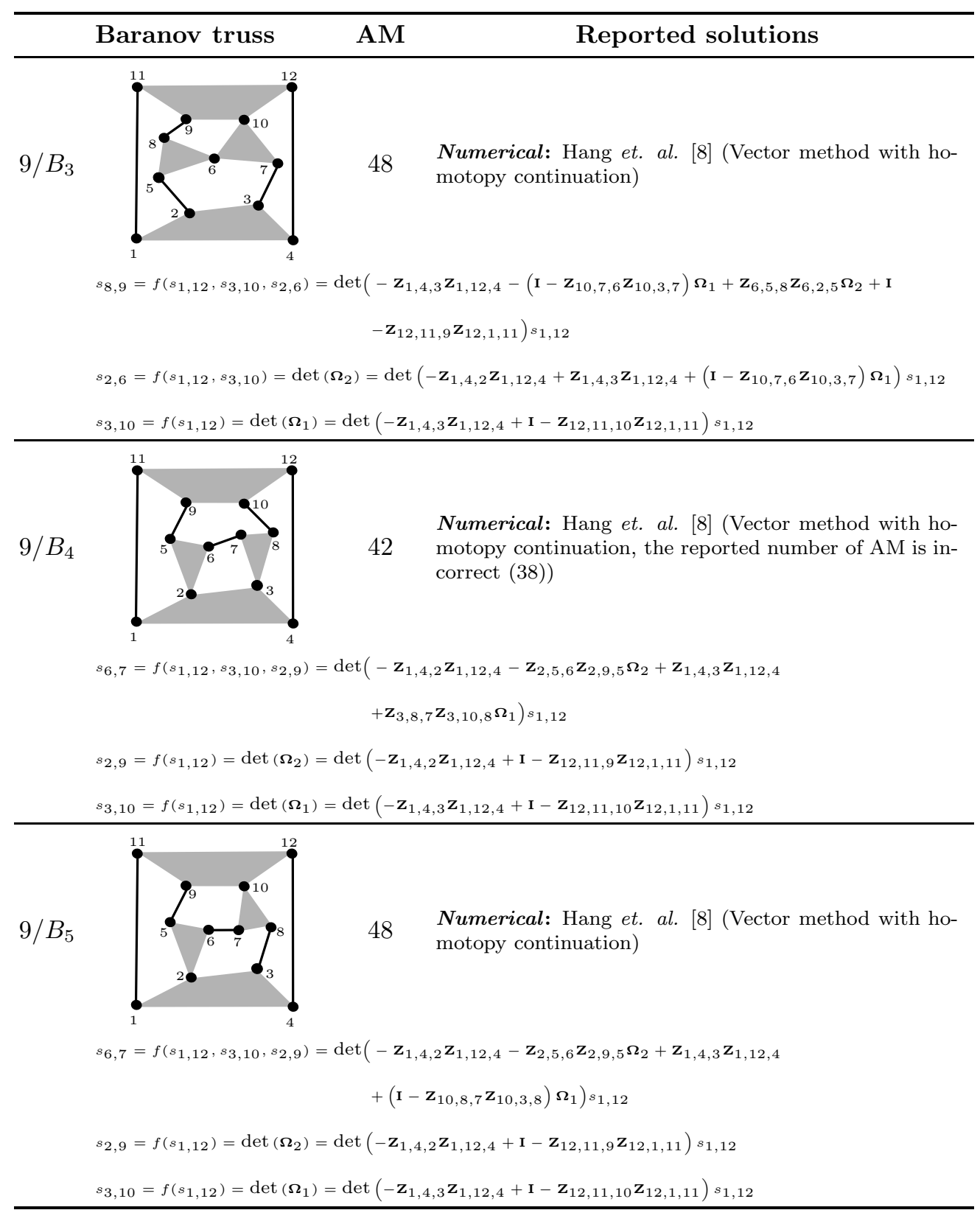

Table 4: Position analysis of all the catalogued Baranov trusses (part 3). 


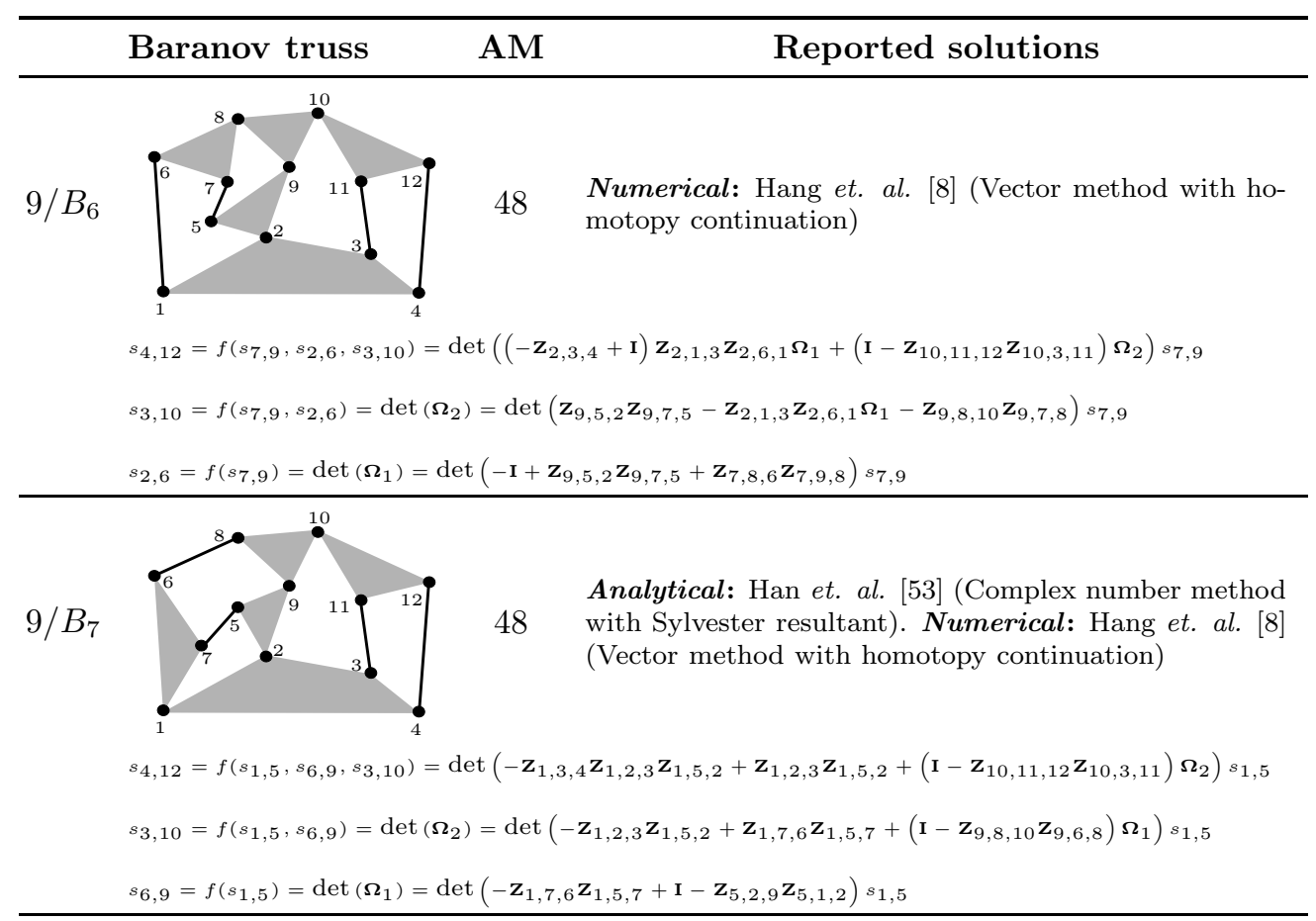
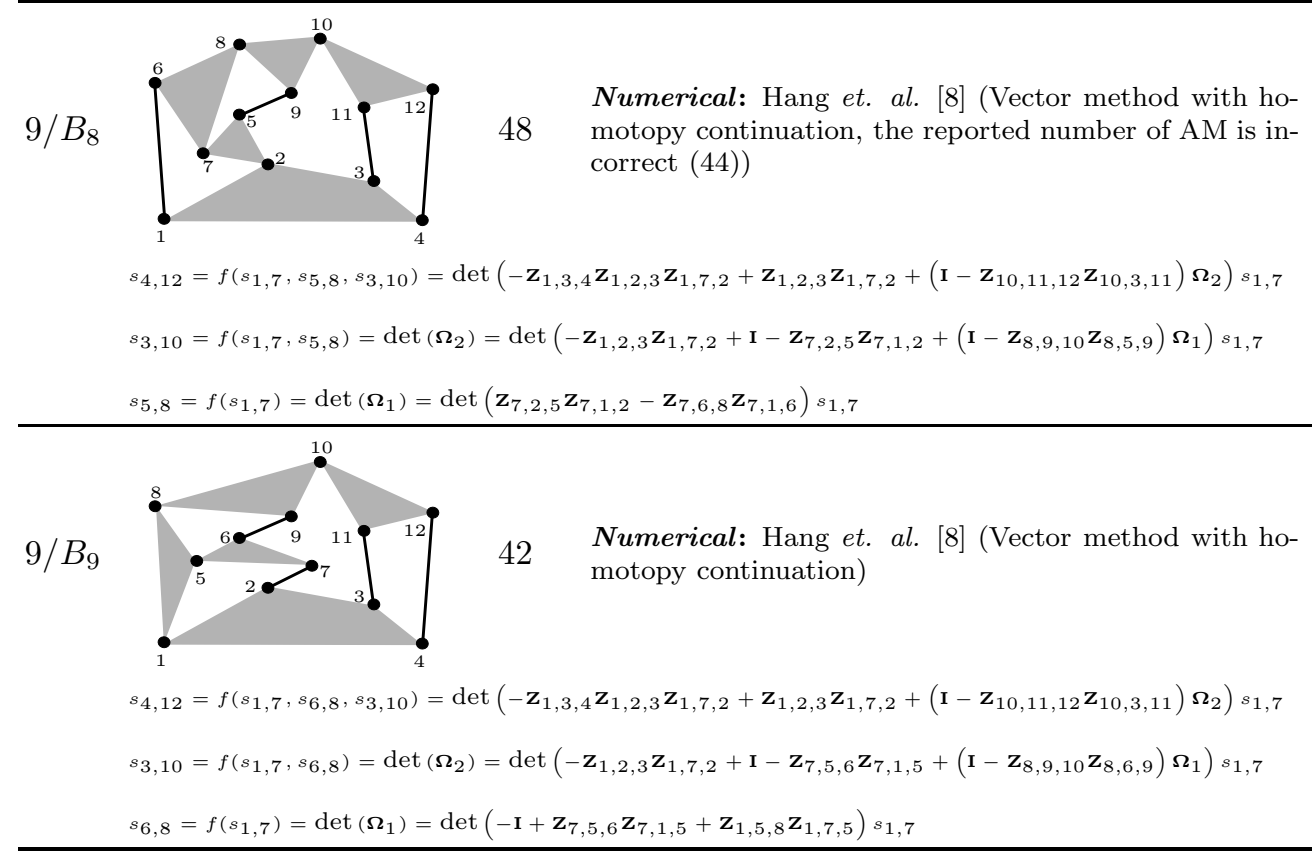

Table 5: Position analysis of all the catalogued Baranov trusses (part 4). 


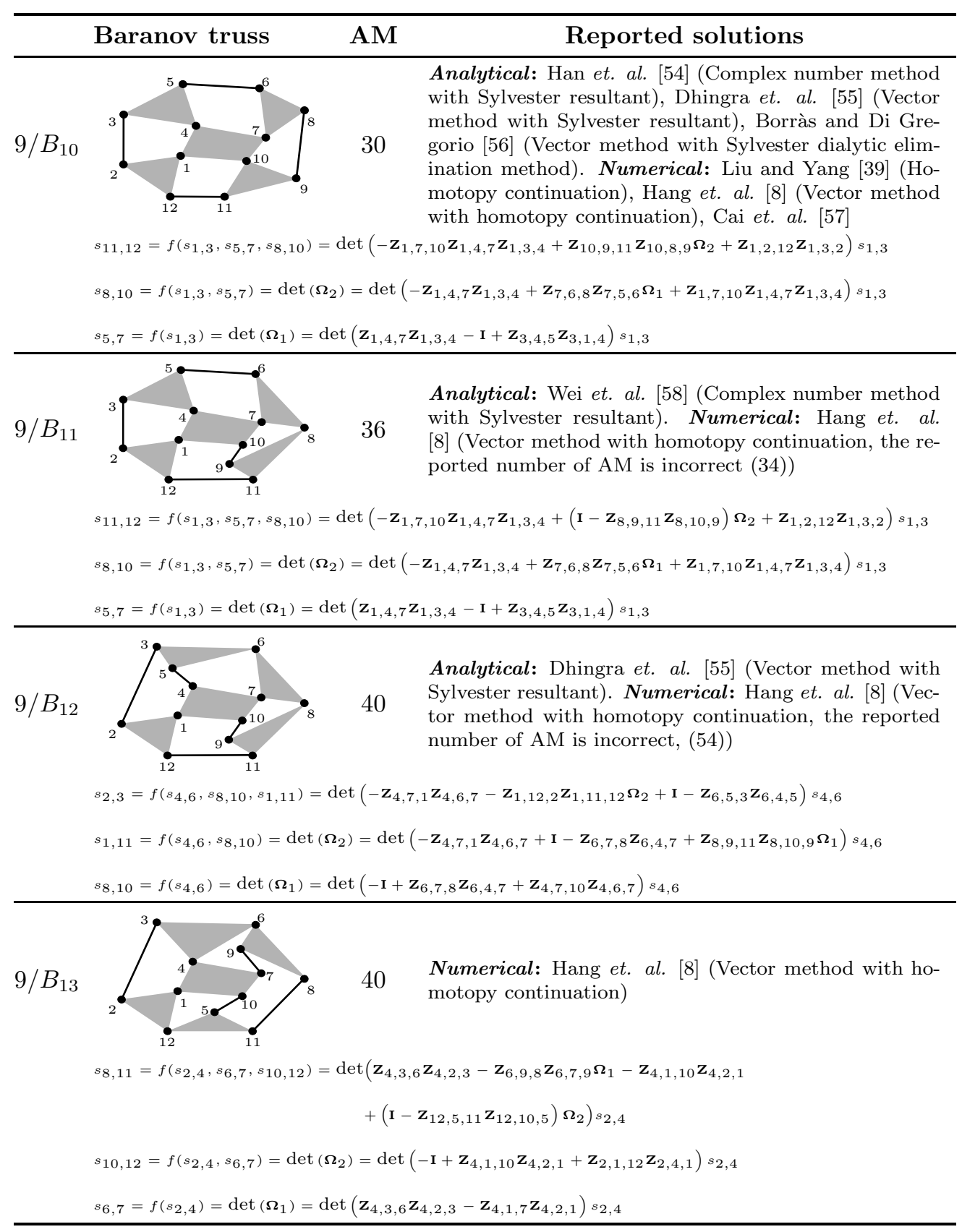

Table 6: Position analysis of all the catalogued Baranov trusses (part 5). 


Baranov truss
AM

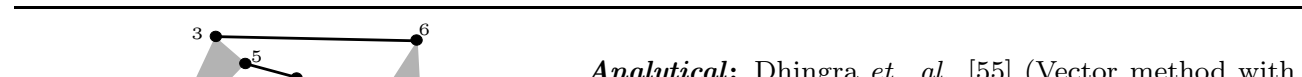

$9 / B_{15}$

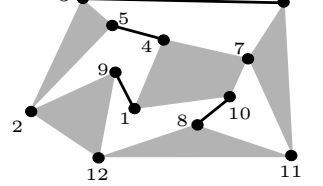

Analytical: Dhingra et. al. 55] (Vector method with Sylvester resultant), Numerical: Hang et. al. 8] (Vector method with homotopy continuation, the reported number of AM is incorrect (26))

$$
\begin{aligned}
& s_{3,6}=f\left(s_{10,11}, s_{1,12}, s_{2,4}\right)=\operatorname{det}\left(-\mathbf{z}_{10,7,1} \mathbf{z}_{10,11,7}-\left(\mathbf{I}-\mathbf{z}_{12,9,2} \mathbf{z}_{12,1,9}\right) \boldsymbol{\Omega}_{1}-\mathbf{z}_{2,5,3} \mathbf{z}_{2,4,5} \boldsymbol{\Omega}_{2}\right. \\
&\left.+\mathbf{I}-\mathbf{z}_{11,7,6} \mathbf{z}_{11,10,7}\right) s_{10,11} \\
& s_{2,4}=f\left(s_{10,11}, s_{1,12}\right)=\operatorname{det}\left(\boldsymbol{\Omega}_{2}\right)=\operatorname{det}\left(-\mathbf{z}_{10,7,1} \mathbf{z}_{10,11,7}-\left(\mathbf{I}-\mathbf{z}_{12,9,2} \mathbf{z}_{12,1,9}\right) \boldsymbol{\Omega}_{1}\right. \\
&\left.+\mathbf{z}_{10,7,4} \mathbf{z}_{10,11,7}\right) s_{10,11}
\end{aligned}
$$

$s_{1,12}=f\left(s_{10,11}\right)=\operatorname{det}\left(\boldsymbol{\Omega}_{1}\right)=\operatorname{det}\left(-\mathbf{z}_{10,7,1} \mathbf{z}_{10,11,7}+\mathbf{I}-\mathbf{Z}_{11,8,12} \mathbf{z}_{11,10,8}\right) s_{10,11}$

Analytical: Han et. al. [60] (Complex number method
with Sylvester resultant). Numerical: Hang et. al. [8]
(Vector method with homotopy continuation)

Analytical: Han et. al. [1] (Complex number method
with Sylvester resultant). Numerical: Hang et. al.
8] (Vector method with homotopy continuation, the re-
ported number of AM is incorrect (66))

Table 7: Position analysis of all the catalogued Baranov trusses (part 6). 


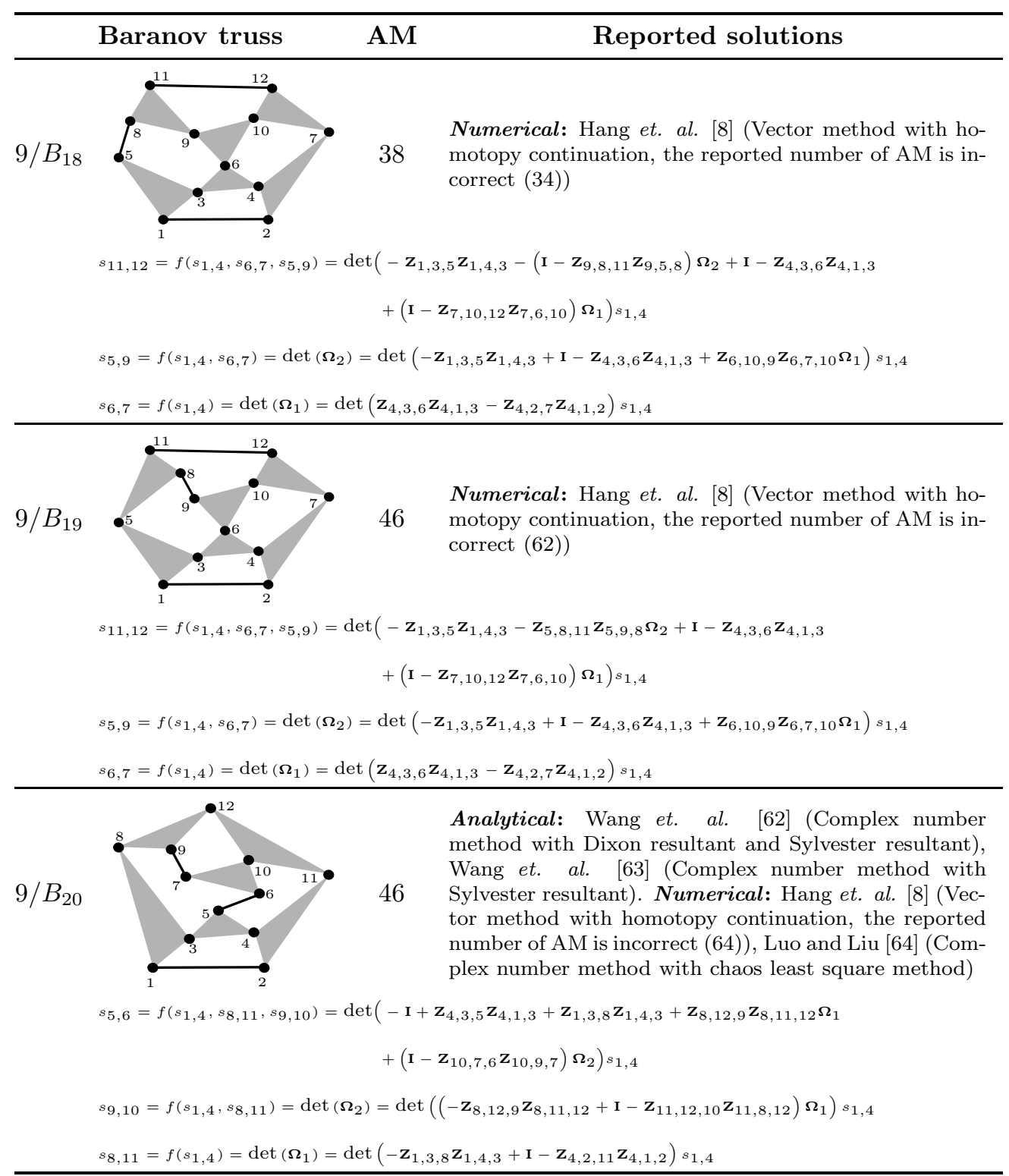

Table 8: Position analysis of all the catalogued Baranov trusses (part 7). 


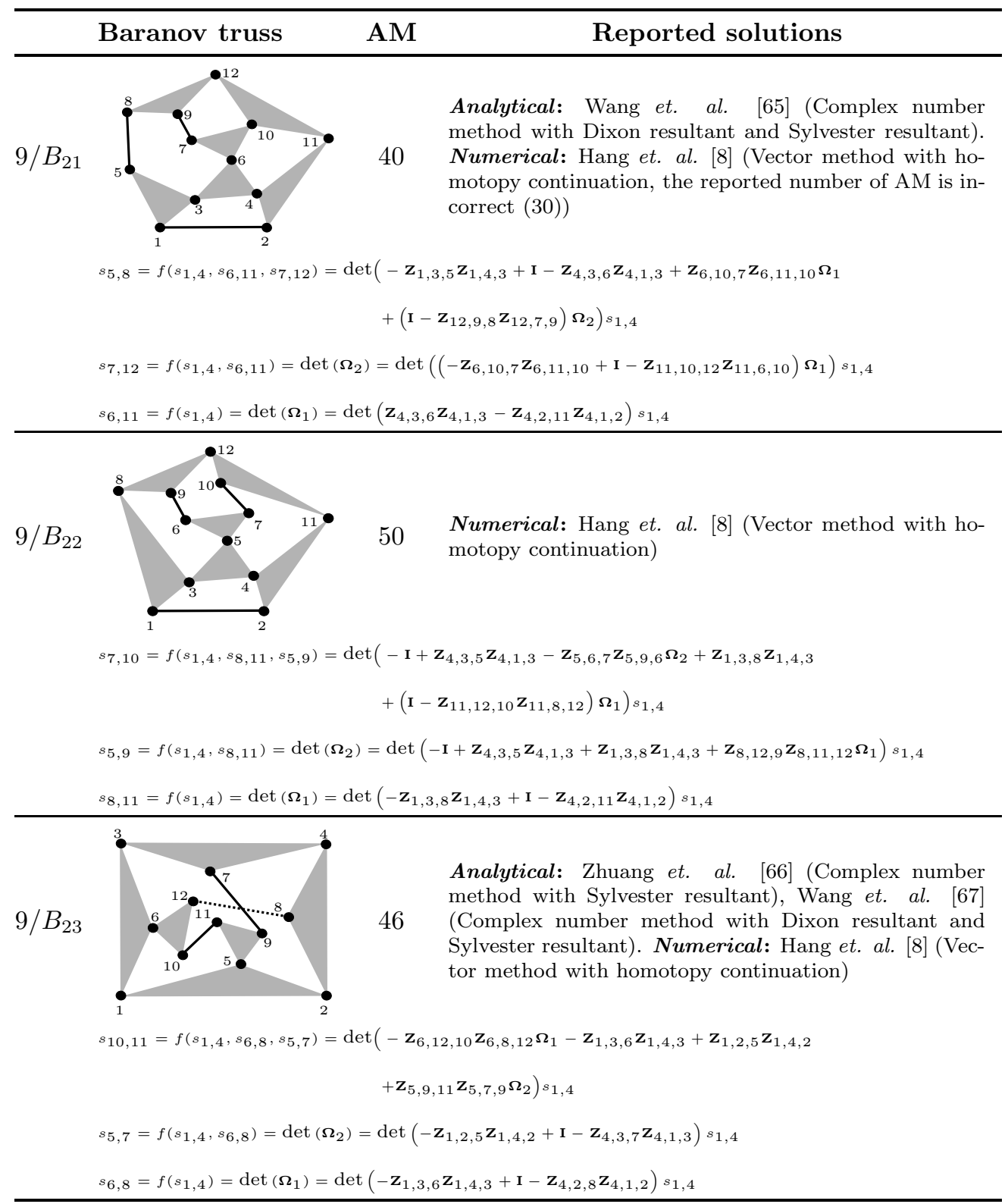

Table 9: Position analysis of all the catalogued Baranov trusses (part 8). 


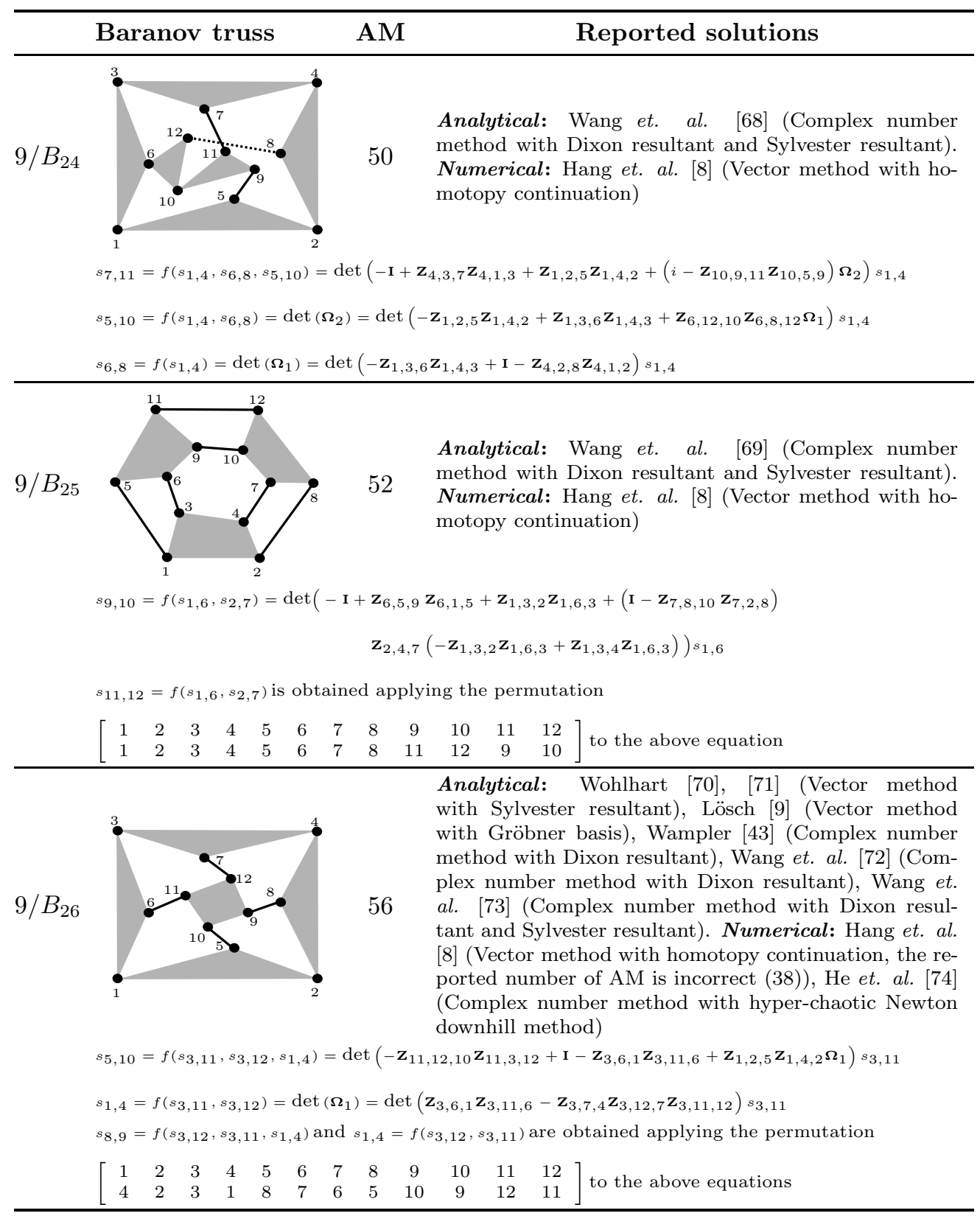

Table 10: Position analysis of all the catalogued Baranov trusses (part 9). 


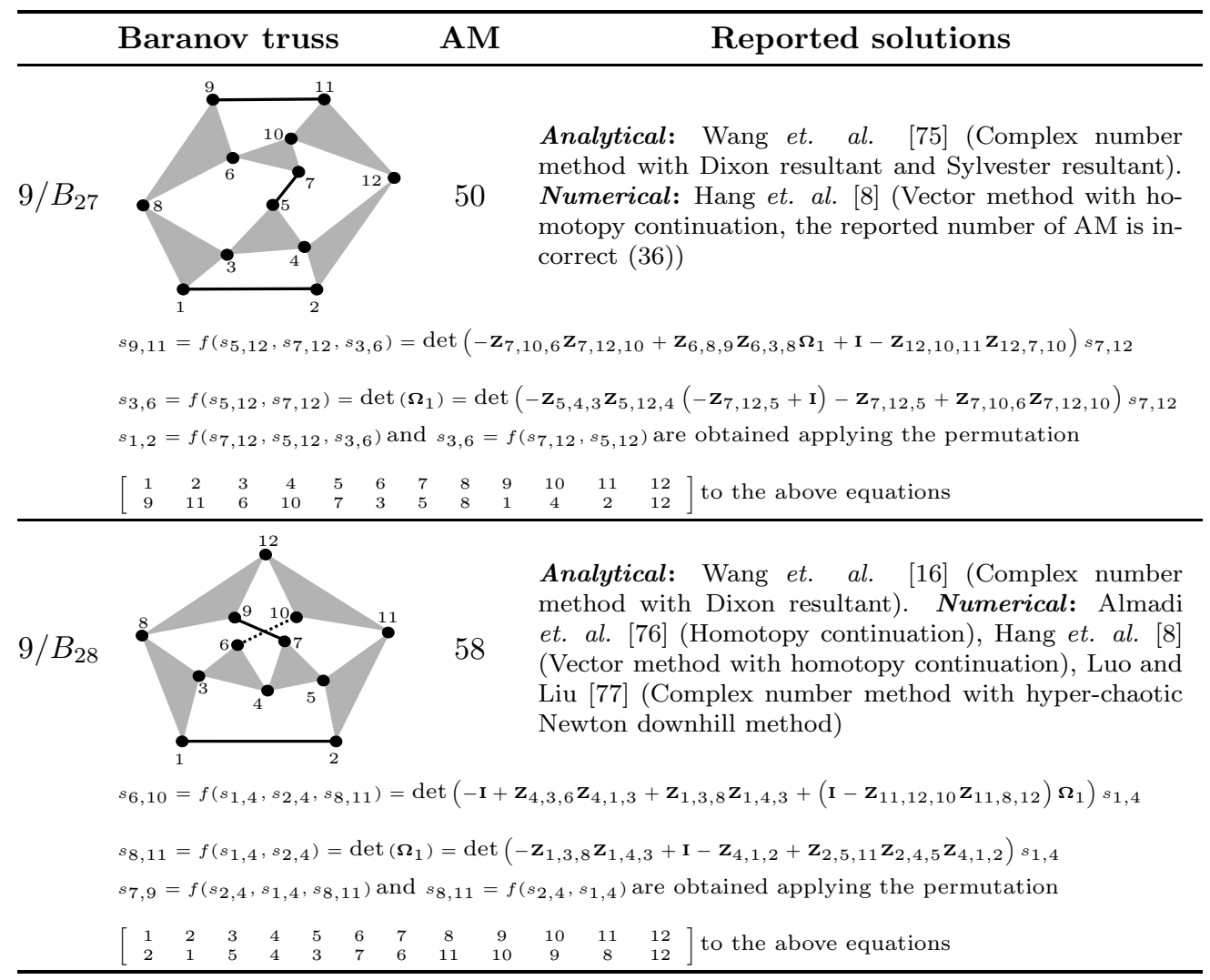

Table 11: Position analysis of all the catalogued Baranov trusses (part 10). 


\section{References}

[1] C. Galletti, A note on modular approaches to planar linkage kinematic analysis, Mechanism and Machine Theory 21 (5) (1986) 385 - 391.

[2] E. Ceresole, P. Fanghella, C. Galletti, Assur's groups, AKCs, basic trusses, SOCs, etc.: Modular kinematics of planar linkages, in: Proceedings of the ASME 1996 International Design Engineering Technical Conferences and Computers in Engineering Conference, Paper No. DETC96/MECH-1027, 1996.

[3] E. Peisach, On Assur groups, Baranov trusses, Grübler chains, planar linkages and on their structural (number) synthesis, in: The 22th Working Meeting of the IFToMM Permanent Commission for Standardization of Terminology, LaMCoS - INSA de LYON, Villeurbanne, France, 2008, pp. 33 -41 .

[4] G. Baranov, Classification, formation, kinematics, and kinetostatics of mechanisms with pairs of the first kind (in russian), in: Proceedings of Seminar on the Theory of Machines and Mechanisms, Moscow, Vol. 2, 1952, pp. $15-39$

[5] N. Manolescu, T. Erdelean, La determination des fermes Baranov avec e=9 elements en utilisant la methode de graphisation inverse, paper d-12, in: Proceedings of the 3rd IFToMM World Congress on the Theory of Machines and Mechanisms, September, Kupari, Yugoslavia, Vol. D, 1971, pp. 177 $-188$.

[6] T. Yang, F. Yao, Topological characteristics and automatic generation of structural synthesis of planar mechanisms based on the ordered single-opened-chains, in: Proceedings of ASME 1994 Mechanisms Conference, Vol. 70, 1994, pp. $67-74$.

[7] N. Manolescu, A method based on Baranov trusses, and using graph theory to find the set of planar jointed kinematic chains and mechanisms, Mechanism and Machine Theory 8 (1) (1973) $3-22$.

[8] L. Hang, Q. Jin, J. Wu, T. Yang, A general study of the number of assembly configurations for multi-circuit planar linkages, Journal of Southeast University (English edition) 16 (1) (2000) 46 51.

[9] S. Lösch, Parallel redundant manipulators based on open and closed normal Assur chains, in: J. Merlet, B. Ravani (Eds.), Computational Kinematics, Kluver Academic Publishers, 1995, pp. $251-260$.

[10] K. Wohlhart, Position analyses of open normal Assur groups a(3.6), in: ASME/IFToMM International Conference on Reconfigurable Mechanisms and Robots, 2009, pp. 88 - 94 .

[11] N. Rojas, F. Thomas, Closed-form solution to the position analysis of Watt-Baranov trusses using the bilateration method, Journal of Mechanisms and Robotics 3 (3) (2011) 031001.

[12] T. Yang, Structural analysis and number synthesis of spatial mechanisms, in: Proceedings of the 6th IFToMM World Congress on the Theory of Machines and Mechanisms, December 15 - 20, New Delhi, India, Vol. 1, 1983, pp. $280-283$.

[13] N. Rojas, F. Thomas, Distance-based position analysis of the three seven-link Assur kinematic chains, Mechanism and Machine Theory 46 (2) (2011) 112 - 126.

[14] J. Graver, Counting on Frameworks: Mathematics to Aid the Design of Rigid Structures, The Mathematical Association of America, 2001.

[15] H. Shen, T. Yang, A numerical method and automatic generation for determining the assemblage configurations of complex planar linkages based on the ordered SOCs, in: Proceedings of ASME 1996 Design Engineering Technical Conferences and Computers in Engineering Conference, Paper No. 96-DETC/MECH-1030, 1996.

[16] P. Wang, Q. Liao, S. Wei, Y. Zuang, Direct position analysis of nine-link Barranov truss based on Dixon resultant, Journal of Beijing University of Aeronautics and Astronautics 32 (7) (2006) 852855,864 .

[17] C. Suh, C. Radcliffe, Kinematics and mechanism design, Wiley, New York, 1978.

[18] C. Galletti, On the position analysis of Assur's groups of high class, Meccanica 14 (1979) 6-10.

[19] J. Chu, W. Cao, T. Yang, Type synthesis of Baranov truss with multiple joints and multiple-joint links, in: Proceedings of ASME 1998 Design Engineering Technical Conferences and Computers in Engineering Conference, Paper No. DETC98/MECH-5972, 1998.

[20] D. Walter, M. Husty, On a nine-bar mechanism, its possible configurations and conditions for flexibility, in: Proceedings of the 12th IFToMM World Congress in Mechanism and Machine Science, June 17 - 20, Besançon, France, 2007.

[21] E. Peisach, Determination of the position of the member of three-joint and two-joint four member Assur groups with rotational pairs (in russian), Machinowedenie (5) (1985) 55 - 61 . 
[22] G. Pennock, D. Kassner, Kinematic analysis of a planar eight-bar linkage: Application to a platformtype robot, Journal of Mechanical Design 114 (1) (1992) 87-95.

[23] C. Gosselin, J. Sefrioui, Polynomial solutions for the direct kinematic problem of planar threedegree-of-freedom parallel manipulators, in: Fifth International Conference on Advanced Robotics, 'Robots in Unstructured Environments', ICAR., 1991, pp. 1124 - 1129 vol.2.

[24] C. Gosselin, J. Sefrioui, M. Richard, Solutions polynomiales au problème de la cinématique des manipulateurs parallèles plans à trois degrés de liberté, Mechanism and Machine Theory 27 (2) (1992) 107-119.

[25] K. Wohlhart, Direct kinematic solution of a general planar Stewart platform, in: Proceedings of the International Conference on Computer Integrated Manufacturing, Zakopane, Poland, 1992, pp. 403-411.

[26] M. Husty, Kinematic mapping of planar three-legged platforms, in: Proceedings of 15th Canadian Congress of Applied Mechanics (CANCAM 1995), Vol. 2, 1995, pp. $876-877$.

[27] X. Kong, C. Gosselin, Forward displacement analysis of third-class analytic 3-RPR planar parallel manipulators, Mechanism and Machine Theory 36 (9) (2001) 1009 - 1018.

[28] C. Collins, Forward kinematics of planar parallel manipulators in the Clifford algebra of p2, Mechanism and Machine Theory 37 (8) (2002) 799 - 813.

[29] L. Han, Y. Zhang, C. Liang, Wu's method for forward displacement analysis of the planar parallel mechanisms, Journal of Beijing University of Aeronautics and Astronautics 24 (1) (1998) 116 - 119.

[30] H. Liu, T. Zhang, H. Ding, Forward solutions of the 3-RPR planar parallel mechanism with Wu's method, Journal of Beijing Institute of Technology 20 (5) (2000) 565 - 569.

[31] Y. Luo, Forward solutions of the 3-RPR planar parallel mechanism with Wu's method, Journal of Hunan University of Arts and Science (Science and Technology) (2) (2004) $27-29$

[32] N. Rojas, F. Thomas, A robust forward kinematics analysis of 3-RPR planar platforms, in: 12th International Symposium on Advances in Robot Kinematics, 2010, pp. $23-32$.

[33] N. Rojas, F. Thomas, The forward kinematics of 3-RPR planar robots: A review and a distancebased formulation, IEEE Transactions on Robotics 27 (1) (2011) 143 - 150.

[34] Y. Luo, X. Li, L. Luo, D. Liao, The research of Newton iterative method based on chaos mapping and its application to forward solutions of the 3-RPR planar parallel mechanism, Machine Design and Research 23 (2) (2007) $37-39$.

[35] R. Chandra, L. Rolland, On solving the forward kinematics of 3RPR planar parallel manipulator using hybrid metaheuristics, Applied Mathematics and Computation In Press, Corrected Proof.

[36] X. Kong, T. Yang, Closed-form displacement analysis of 3-loop Baranov trussed, in: Proceedings of International Conference on Spatial Mechanisms and High Class Mechanisms (Theory and Practice), 1994.

[37] C. Innocenti, Position analysis in analytical form of the 7-link Assur kinematic chain featuring one ternary link connected to ternary links only, Mechanism and Machine Theory 32 (4) (1997) 501509.

[38] A. Dhingra, A. Almadi, D. Kohli, Closed-form displacement analysis of 8, 9 and 10-link mechanisms: Part I: 8-link 1-DOF mechanisms, Mechanism and Machine Theory 35 (6) (2000) $821-850$

[39] A. Liu, T. Yang, Displacement analysis of planar complex mechanisms using continuation method, Mechanical Science and Technology 13 (2) (1994) $55-62$.

[40] C. Innocenti, Analytical-form position analysis of the 7-link Assur kinematic chain with four seriallyconnected ternary links, Journal of Mechanical Design 116 (2) (1994) 622-628.

[41] A. Almadi, A. Dhingra, D. Kohli, Closed-form displacement analysis of SDOF 8 link mechanisms, in: Proceedings of ASME 1996 Design Engineering Technical Conferences and Computers in Engineering Conference, Paper No. 96-DETC/MECH-1206, 1996.

[42] A. Dhingra, A. Almadi, D. Kohli, A Gröbner-Sylvester hybrid method for closed-form displacement analysis of mechanisms, Journal of Mechanical Design 122 (4) (2000) 431-438.

[43] C. Wampler, Solving the kinematics of planar mechanisms by Dixon determinant and a complexplane formulation, Journal of Mechanical Design 123 (3) (2001) 382-387.

[44] H. Shen, K. Ting, T. Yang, Configuration analysis of complex multiloop linkages and manipulators, Mechanism and Machine Theory 35 (3) (2000) 353 - 362.

[45] C. Innocenti, Analytical determination of the intersections of two coupler-point curves generated by two four-bar linkages, in: Computational Kinematics, J. Angeles, G. Hommel, P. Kovács (Eds.), Kluwer Academic Publishers, Dordrecht, the Netherlands, 1993, pp. $251-262$.

[46] C. Innocenti, Polynomial solution to the position analysis of the 7-link Assur kinematic chain with one quaternary link, Mechanism and Machine Theory 30 (8) (1995) 1295 - 1303.

[47] L. Han, Q. Liao, C. Liang, The closed form displacement analysis of a seven-link Barranov-truss 
and all the Assur groups connected with it, Mechanical Science and Technology 17 (5) (1998) 785 -788 .

[48] P. Wang, Q. Liao, S. Wei, Forward didplacement analysis of a seven-link Barravo truss based on Wu method, Mechanical Science and Technology 25 (6) (2006) $748-752$.

[49] Z. Ni, Q. Liao, S. Wei, New research of forward displacement analysis of 6-link Assur group, in: 2nd International Conference on Information Science and Engineering (ICISE), 2010, pp. 5187 -5190.

[50] A. Dhingra, A. Almadi, D. Kohli, Closed-form displacement and coupler curve analysis of planar multi-loop mechanisms using Gröbner bases, Mechanism and Machine Theory 36 (2) (2001) 273 298

[51] S. Wei, X. Zhou, Q. Liao, Research on assembly configurations for nine-link Barranov truss, Mechanical Science and Technology 23 (8) (2004) $962-965$.

[52] K. Wohlhart, Robots based on Assur group a(3.5), in: J. Lenarcic, P. Wenger (Eds.), Advances in Robot Kinematics: Analysis and Design, Springer Netherlands, 2008, pp. 165-175.

[53] L. Han, Q. Liao, C. Liang, Closed-form displacement analysis for a nine-link Barranov truss or a eight-link Assur group, Mechanism and Machine Theory 35 (3) (2000) $379-390$.

[54] L. Han, Q. Liao, C. Liang, A kind of algebraic solution for the position analysis of a planar basic kinematic chain, Journal of Machine Design 16 (3) (1999) 16 - 18.

[55] A. Dhingra, A. Almadi, D. Kohli, Closed-form displacement analysis of 10-link 1-DOF mechanisms: Part 2 - polynomial solutions, Mechanism and Machine Theory 36 (1) (2001) 57 - 75.

[56] J. Borràs, R. D. Gregorio, Polynomial solution to the position analysis of two Assur kinematic chains with four loops and the same topology, Journal of Mechanisms and Robotics 1 (2) (2009) 021003.

[57] S. Cai, Y. Zuo, N. Chen, Position analysis of planar complex mechanisms using triangular vector loop method, Journal of Lanzhou University of Technology 31 (5) (2005) 35 - 38.

[58] S. Wei, X. Zhou, Q. Liao, Closed-form displacement analysis of one kind of nine-link Barranov trusses, in: Proceedings of the 11th IFToMM World Congress in Mechanism and Machine Science, April 1-4, Tianjin, China, 2004, pp. 1176 - 1177.

[59] L. Han, Q. Liao, C. Liang, Closed-form displacement analysis of a planar fifth-class group with lower pairs, Journal of Beijing University of Aeronautics and Astronautics 24 (5) (1998) 603 - 606.

[60] L. Han, Q. Liao, C. Liang, Closed-form displacement analysis of an eight links planer Assur group, in: Proceedings of the 10th World Congress on the Theory of Machine and Mechanisms, June 20-24, Oulu, Finland, 1999, pp. $168-173$

[61] L. Han, Q. Liao, C. Liang, Complex number method for kinematic analysis of planar Assur groups, Mechanical Science and Technology 17 (3) (1998) 410 - 412.

[62] P. Wang, Q. Liao, Y. Zhuang, S. Wei, Direct position analysis of a nine-link Barranov truss, Journal of Tsinghua University (Science and Technology) 46 (8) (2006) 1373 - 1376.

[63] P. Wang, Q. Liao, S. Wei, Y. Zhuang, Displacement analysis of a kind of nine-link Barranov truss, Journal of Beijing University of Posts and Telecommunications 29 (3) (2006) $12-17$.

[64] Y. Luo, Q. Liu, Forward displacement analysis of 25th nine-link Barranov truss based on chaos least square method, in: 2nd International Conference on Computer Modeling and Simulation (ICCMS), Vol. 1, 2010, pp. $184-188$.

[65] P. Wang, Q. Liao, Z. Lu, Displacement analysis of a nine-link Barranov truss, Machine Design and Research 25 (2) (2009) $33-36$.

[66] Y. Zhuang, P. Wang, Q. Liao, Research on displacement analysis of a kind of non-planar nine link Barranov truss, Journal of Beijing University of Posts and Telecommunications 29 (6) (2006) 13 16.

[67] P. Wang, L. Wang, D. Li, J. Gu, J. Song, Closed-form displacement analysis of a kind of non-planar nine-link Barranov truss, in: IEEE International Conference on Automation and Logistics, 2008. ICAL 2008., 2008, pp. $2397-2401$.

[68] P. Wang, Q. Liao, Z. Lu, Displacement analysis of non-planar nine-link Barranov truss, Journal of Beijing University of Posts and Telecommunications 31 (4) (2008) $10-14$

[69] P. Wang, Q. Liao, Y. Zuang, S. Wei, Displacement analysis of nine-link Barranov truss, Chinese Journal of Mechanical Engineering 43 (7) (2007) 11 - 15.

[70] K. Wohlhart, Position analysis of the rhombic Assur group 4.4, in: Proceedings of the RoManSy, CISM- IFTOMM Symposium, Gdansk, Poland, 1994, pp. $21-31$.

[71] K. Wohlhart, Position analyses of normal quadrilateral Assur groups, Mechanism and Machine Theory 45 (9) (2010) $1367-1384$.

[72] P. Wang, Q. Liao, S. Wei, Y. Zuang, Forward displacement analysis of a kind of nine-link Barranov truss based on Dixon resultants, China Mechanical Engineering 17 (21) (2006) 2034 - 2038. 
[73] P. Wang, Q. Liao, Y. Zhuang, S. Wei, A method for position analysis of a kind of nine-link Barranov truss, Mechanism and Machine Theory 42 (10) (2007) 1280 - 1288.

[74] Z. He, Y. Luo, Q. Liu, Forward displacement analysis of a kind of nine-link Barranov truss based on hyper-chaotic Newton downhill method, in: 2nd International Conference on Intelligent HumanMachine Systems and Cybernetics (IHMSC), Vol. 2, 2010, pp. $137-142$.

[75] P. Wang, Q. Liao, Y. Zhuang, S. Wei, Research on position analysis of a kind of nine-link Barranov truss, Journal of Mechanical Design 130 (1) (2008) 011005.

[76] A. Almadi, A. Dhingra, D. Kohli, Displacement analysis of ten-link kinematic chains using homotopy, in: Proceedings of 9th World Congress on Theory of Machines and Mechanisms, Vol. 1, 1995, pp. $90-94$.

[77] Y. Luo, Q. Liu, Forward displacement analysis of non-plane two coupled degree nine-link Barranov truss based on hyper-chaotic Newton downhill method, Applied Mechanics and Materials 20 - 23 (2010) 659-664. 